\title{
Adaptive Tracking of Angular Velocity for a Planar Rigid Body With Unknown Models for Inertia and Input Nonlinearity
}

\author{
Nalin A. Chaturvedi, Amit K. Sanyal, Madhusudhan Chellappa, Jean Luc Valk, N. Harris McClamroch, Fellow, IEEE,
} and Dennis S. Bernstein, Fellow, IEEE

\begin{abstract}
The problem of a planar rigid body, with unknown rotational inertia and an unknown input nonlinearity, tracking a desired angular velocity trajectory is addressed using adaptive feedback control. First, an adaptive controller is developed for tracking a desired angular velocity command, assuming linearly entering control. Sufficient conditions on the command signal for estimating the inertia are given. To account for an unknown input nonlinearity, a piecewise-linear approximation of the nonlinearity is inverted to obtain improved angular velocity tracking and inertia identification. Finally, a direct adaptive algorithm, incorporating feedback linearization is proposed, and Lyapunov analysis is used to show convergence of the angular velocity and inertia estimate errors. The approach is validated by experimental implementation.
\end{abstract}

Index Terms-Adaptive control, angular velocity tracking, generalized solution, input nonlinearity, rotating bodies.

\section{INTRODUCTION}

$\mathbf{T}$ HE rotational control of a rigid body in three dimensions is a widely studied and fundamental problem in spacecraft dynamics [1]-[6]. Minimum fuel and minimum time performance have been studied in [7] and [8], while stabilization of multiple bodies has been studied in [9] and [10]. Although it is generally assumed that the spacecraft mass distribution is known, there are limitations in practice on the ability to determine the exact mass distribution due to fuel usage, moving appendages, and complex geometry. Hence, it is of interest to develop controllers that can operate reliably with as little inertia modeling as possible. Adaptive tracking for a three-dimensional (3-D) rigid body without inertia modeling is considered in [11].

Another common assumption is the affine (linearly entering) nature of the control input. While adaptive control of affine control systems has been widely studied, see for example [12]-[14], there is relatively little in the literature on adaptive controllers for nonaffine systems. Recent results in the literature have addressed the above problems under various assumptions [15]-[18]. The results in these papers involve inversion of the input nonlinearity assuming that the partial derivative of the input nonlinearity is bounded away from zero with a constant sign; this assumption guarantees that there exists a local inverse.

Manuscript received June 25, 2004; revised July 14, 2005. Manuscript received in final form February 23, 2006. Recommended by Associate Editor R. S. Erwin. This work was supported in part by the Air Force Office of Scientific Research under Grant F49620-98-1-0037 and by the National Science Foundation under Grant ECS-0140053 and Grant ECS-0244977.

The authors are with the Department of Aerospace Engineering, University of Michigan, Ann Arbor, MI 48109-2140 USA (e-mail: dsbaero@umich.edu).

Digital Object Identifier 10.1109/TCST.2006.876628
In the present paper, we develop an adaptive controller, along with simulation and experimental results, for an adaptive control problem involving a model of a planar rigid body that is nonaffine in the input. Our approach involves two steps. First, we ignore the input nonlinearity and address the inertia-uncertainty problem by deriving an adaptive controller that guarantees that the angular velocity asymptotically tracks an angular velocity command without any information concerning the mass distribution of the rigid body. This controller provides asymptotic tracking of a large class of angular velocity commands. This adaptive controller has the form of a PI control law. The integrator state, which corresponds to the inertia estimate, is shown to converge to the actual inertia under persistent excitation.

Next, we include the uncertain input nonlinearity. By parameterizing the input nonlinearity we develop an adaptive feedback linearization controller. Using Lyapunov techniques, we obtain globally convergent tracking for a large class of command signals. Simulation results are given to demonstrate the effectiveness of the adaptive controller.

Next, we implement the adaptive feedback linearization controller on an experimental testbed. To further compare the performance of this controller with other techniques, we compare the experimental results with an adaptive controller that does not take into account the nonlinearity of the input and also, with an adaptive controller that uses an approximation of the inverse of the input nonlinearity. We also discuss the problem of bursting, which might occur in such nonlinear adaptive control systems.

Our development also highlights existence and uniqueness issues that arise due to inversion of the input nonlinearity. In particular, we show that the input nonlinearity, coupled with the adaptive controller, may lead to discontinuous closed-loop dynamics. We state assumptions under which the closed-loop has a solution. The notion of solution of the closed-loop in these cases is usually that of a generalized solution, in the sense of Filippov [19] or Krasovskii [20].

The contents of this paper are as follows. In Section II, we introduce the problem of adaptive control for a rigid planar rotating body that has an input nonlinearity and present the first step in our development of an adaptive controller. We design an adaptive control scheme that provides angular velocity tracking for a planar rigid body with unknown inertia. In addition, we present and illustrate a method for identifying the unknown inertia. Furthermore, we show global tracking and parameter convergence for command signals that are not necessarily bounded. Next, in Section III, we extend the adaptive con- 
troller to include an unknown input nonlinearity. In Section IV, Lyapunov's analysis is used to guarantee asymptotic tracking of the angular velocity command. A description of the testbed and control hardware used for experiments is presented in Section V. In Section VI, we present experimental and simulation results for single-degree-of-freedom rigid body rotation for three different controllers: a nonadaptive proportional controller, an adaptive controller designed to adapt to the unknown inertia only assuming known linear input, and an adaptive controller that adapts to unknown inertia only with piecewise linear approximation of the inverse of the input nonlinearity. Finally, in Section VII, the performance of the adaptive feedback linearization controller is validated by simulations and experimental implementation.

\section{AdAPtive Control With An AfFine Control InPUT}

In this section, we introduce the planar rigid body model with nonaffine input and we define the adaptive control performance objectives. Subsequently, we consider the first step in the development of a controller structure, wherein we design an adaptive controller that adapts to the unknown inertia but ignores the input nonlinearity.

Consider a rigid body constrained to rotate about a fixed axis. For $t \geq 0$, the equation of motion is given by

$$
\dot{\omega}=\frac{1}{J} \tau
$$

where $\omega$ is the angular velocity of the body about its axis of rotation, $J$ is the moment of inertia of the body about its axis of rotation, and $\tau$ is the applied torque. We assume that $J$ is positive but is otherwise unknown. Furthermore, $\tau=h(u)$ where $h: \mathbb{R} \mapsto \mathbb{R}$ is a continuous onto function of the control input $u$. Hence, we can write (1) as

$$
\dot{\omega}=\frac{1}{J} h(u)
$$

Remark 1: The fact that $h$ is onto guarantees that a control torque of any magnitude can be generated. However, we do not require the function $h$ to be one-to-one. Thus, there can be multiple values of $u$ that generate the same torque $\tau=h(u)$.

Let $\nu:[0, \infty) \mapsto \mathbb{R}$ denote the angular velocity command. The control objective is to design an adaptive controller that can track a large class of command signals $\nu$ without knowledge of $J$ and the input nonlinearity $h(\cdot)$.

We first consider the design of an adaptive controller for the associated affine system, that is, for the case $h(u)=u$. The results proved in this section are extended to the nonaffine case in Sections III and IV.

Defining the angular velocity error $\tilde{\omega} \triangleq \omega-\nu$, it follows from (1) that $\tilde{\omega}$ satisfies

$$
\dot{\tilde{\omega}}=-\dot{\nu}+\frac{1}{J} u
$$

The control objective is to determine $u$ such that $\tilde{\omega}(t) \rightarrow 0$ as $t \rightarrow \infty$ for all initial conditions $\omega(0)$ and without knowledge of $J$. The following result provides an adaptive controller for angular velocity based on an estimate $\hat{J}(t)$ of $J$. We denote the error in the inertia estimate by $\tilde{J} \triangleq \hat{J}-J$.
Theorem 1: Assume that $\nu$ is continuous and $\dot{\nu}$ is piecewise $C^{1}$ and bounded. Let $k>0$ and $q>0$, and consider the adaptative control law

$$
\begin{aligned}
& \dot{\hat{J}}=-q \dot{\nu} \tilde{\omega} \\
& \tau=-k \tilde{\omega}+\dot{\nu} \hat{J}
\end{aligned}
$$

and the resulting closed-loop dynamics (3), (4), and (5) in error coordinates given by

$$
\begin{aligned}
& \dot{\tilde{\omega}}=-\frac{k}{J} \tilde{\omega}+\frac{\dot{\nu}}{J} \tilde{J} \\
& \dot{\tilde{J}}=-q \dot{\nu} \tilde{\omega} .
\end{aligned}
$$

Then, the zero equilibrium solution of (6) and (7) is Lyapunov stable and satisfies $\tilde{\omega}(t) \rightarrow 0$ as $t \rightarrow \infty$ for all $\omega(0)$ and $\hat{J}(0)$. Furthermore, $\lim _{t \rightarrow \infty} \dot{\tilde{J}}(t)=0$ and $\lim _{t \rightarrow \infty} \hat{J}(t)$ exists.

Proof: Using (3), (4), and $\stackrel{t \rightarrow \infty}{(5)}$, we obtain the linear timevarying system given by (6) and (7). Since $\dot{\nu}$ is piecewise $C^{1}$ and bounded, the right-hand side of (6) and (7) is piecewise $C^{1}$ in time and globally Lipschitz in $\tilde{\omega}$ and $\tilde{J}$, uniformly in time. Furthermore, $[\tilde{\omega} \tilde{J}]=\left[\begin{array}{ll}0 & 0\end{array}\right]$ is an equilibrium of (6) and (7).

To prove asymptotic tracking, consider the positive-definite Lyapunov candidate

$$
V(\tilde{\omega}, \tilde{J}) \triangleq \frac{1}{2}\left(J \tilde{\omega}^{2}+\frac{1}{q} \tilde{J}^{2}\right)
$$

which does not depend explicitly on time and is radially unbounded. The derivative of $V$ along the trajectories of the closed-loop system is given by

$$
\dot{V}(\tilde{\omega}, \tilde{J})=-k \tilde{\omega}^{2}+\dot{\nu} \tilde{J} \tilde{\omega}+\frac{1}{q} \tilde{J} \dot{\tilde{J}}=-k \tilde{\omega}^{2}
$$

which shows that $\dot{V}$ is negative semidefinite and is not an explicit function of time. Theorem 8.4 of [21] then implies that, for all initial conditions $\tilde{\omega}(0)$ and $\tilde{J}(0)$, the solutions of (6) and (7) are bounded and approach the set $E \triangleq \dot{V}^{-1}(0)=\{[\tilde{\omega} \tilde{J}] \in$ $\left.\mathbb{R}^{2}: \tilde{\omega}=0\right\}$. Hence, $\tilde{\omega}(t) \rightarrow 0$ as $t \rightarrow \infty$. Since $V$ is globally positive definite and radially unbounded and $\dot{V}$ is negative semidefinite, it follows that the system (6) and (7) is Lyapunov stable.

Since $\tilde{\omega}(t) \rightarrow 0$ as $t \rightarrow \infty$ and $\dot{\nu}$ is bounded, it follows from (7) that $\dot{\tilde{J}}(t) \rightarrow 0$ and, thus $\dot{\hat{J}}(t) \rightarrow 0$ as $t \rightarrow \infty$. Furthermore, $\tilde{\omega}(t)$ and $\tilde{J}(t)$, and, hence, $\hat{J}(t)$, are bounded. Now, since $\dot{V}(t) \triangleq \dot{V}(\tilde{\omega}(t), \tilde{J}(t)) \leq 0$ and $V(t) \triangleq V(\tilde{\omega}(t), \tilde{J}(t)) \geq 0$ for all $t \geq 0$, it follows that $\lim _{t \rightarrow \infty} V(t)$ exists.

Next, (8) can be rewritten along the solutions of (6) and (7) as

$$
\tilde{J}^{2}(t)=q\left[2 V(t)-J \tilde{\omega}^{2}(t)\right] .
$$

Since $\lim _{t \rightarrow \infty} V(t)$ exists and $\lim _{t \rightarrow \infty} \tilde{\omega}(t)=0$, it follows from (10) that

$$
\lim _{t \rightarrow \infty} \tilde{J}^{2}(t)=2 q \lim _{t \rightarrow \infty} V(t) .
$$

Therefore, since $\tilde{J}(t)$ is continuous, $\lim _{t \rightarrow \infty} \tilde{J}(t)$ exists. Hence, $\lim _{t \rightarrow \infty} \hat{J}(t)$ exists. 
Note that the control law (4) does not require knowledge of the inertia $J$. Although $\dot{\hat{J}}$ converges to zero and $\hat{J}$ converges, $\hat{J}$ does not necessarily converge to the actual inertia $J$. We now give a sufficient condition under which $\hat{J}$ converges to $J$. Lemma 1 in Appendix A is needed.

Theorem 2: Consider the closed-loop system consisting of (3) and the adaptive control law (4) and (5), where $k>0$ and $q>0$. Assume that $\dot{\nu}$ is piecewise continuous and bounded and either $\lim _{t \rightarrow \infty} \dot{\nu}(t) \neq 0$ or $\lim _{t \rightarrow \infty} \dot{\nu}(t)$ does not exist. Then, $\hat{J}(t) \rightarrow J$ as $t \rightarrow \infty$ for all $\omega(0)$ and $\hat{J}(0)$.

Proof: Theorem 1 implies that $\tilde{\omega}(t) \rightarrow 0$ as $t \rightarrow \infty$. Then, $\tilde{\omega}(t)-\tilde{\omega}(0)=\int_{0}^{t} \dot{\tilde{\omega}}(t) d t$ implies that

$$
\lim _{t \rightarrow \infty} \int_{0}^{t} \dot{\tilde{\omega}}(t) d t=-\tilde{\omega}(0) .
$$

Since $\dot{\nu}$ is piecewise continuous and bounded, (6) implies that $\dot{\tilde{\omega}}(t)$ is globally piecewise uniformly continuous. Now, applying the generalized version of Barbalat's Lemma given in Lemma 2 in Appendix B, yields $\lim _{t \rightarrow \infty} \dot{\tilde{\omega}}(t)=0$. Therefore, it follows from (6) that

$$
\lim _{t \rightarrow \infty} \dot{\nu}(t) \tilde{J}(t)=0 .
$$

Since, by Theorem 1, $\lim _{t \rightarrow \infty} \tilde{J}(t)$ exists, and either $\lim _{t \rightarrow \infty} \dot{\nu}(t) \neq 0$ or $\lim _{t \rightarrow \infty} \dot{\nu}(t)$ does not exist, we obtain the result from Lemma 1 in Appendix A, that $\tilde{J}(t)$ converges to zero.

Note that Theorems 1 and 2 require that $\dot{\nu}$ be piecewise $C^{1}$ and bounded. However, $\nu$ need not be bounded.

Example 1: Consider the angular velocity command

$$
\nu(t)= \begin{cases}0, & 0 \leq t<10 \\ 0.1 t-1, & 10 \leq t<30 \\ 2, & 30 \leq t<\infty\end{cases}
$$

Note that $\dot{\nu}(t)$ is piecewise continuous and bounded. The inertia of the planar rotating body is taken to be $J=20.0 \mathrm{~kg} \cdot \mathrm{m}^{2}$, and its initial estimate is $\hat{J}(0)=15.0 \mathrm{~kg} \cdot \mathrm{m}^{2}$. Let $k=10$ and $q=$ 2.8. The initial angular velocity error is given by $\tilde{\omega}(0)=1 \mathrm{rad} / \mathrm{s}$. The angular velocity tracking error, inertia estimate error, and applied input torque are shown in Fig. 1. Fig. 1 shows that $\hat{J}$ converges but does not converge to $J$. In fact, $\dot{\nu}$ does not satisfy the assumptions of Theorem 2. However, $\tilde{\omega}$ converges to zero. The torque is seen to have an initial transient. The torque at time zero $\tau(0)=-k \tilde{\omega}(0)+\dot{\nu}(0) \hat{J}(0)=-10 \mathrm{~N} \cdot \mathrm{m}$, where $\dot{\nu}(0)=0 \mathrm{rad} / \mathrm{s}^{2}$.

Example 2: Consider two command signals that satisfy the conditions given in Theorem 2, namely

$$
\nu(t)= \begin{cases}0, & 0 \leq t<10 \\ t-10, & 10 \leq t<\infty\end{cases}
$$

and

$$
\nu(t)= \begin{cases}5(t-2\lfloor t / 2\rfloor), & 2 n \leq t<2 n+1 \\ 5(2-t+2\lfloor t / 2\rfloor), & 2 n+1<t \leq 2 n+2\end{cases}
$$

where $n$ is a nonnegative integer. Both signals are piecewise $C^{1}$, and the first one is unbounded. The initial condition for $\hat{J}$ and
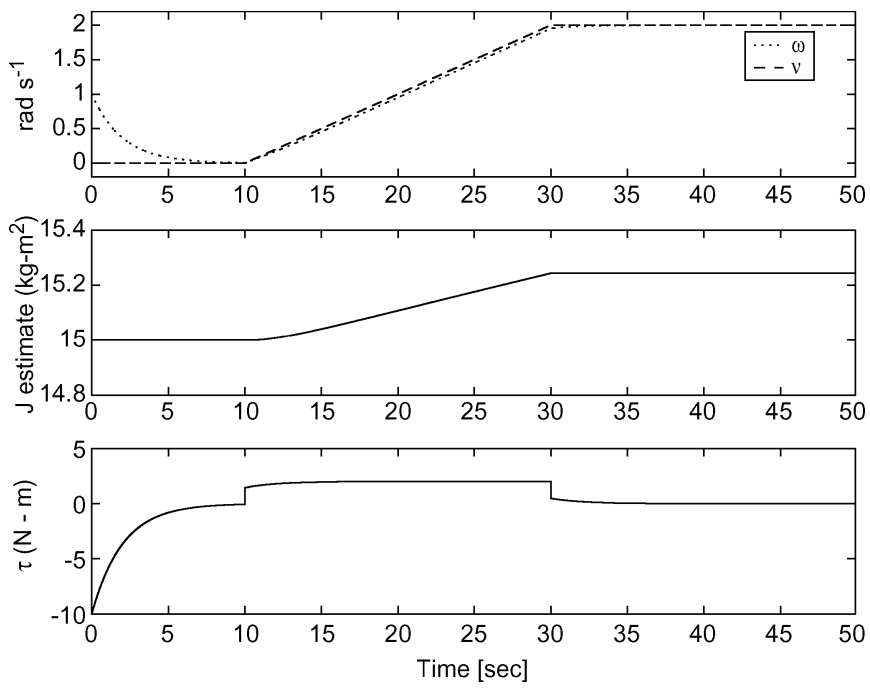

Fig. 1. Angular velocity, inertia estimate, and control torque for Example 1.
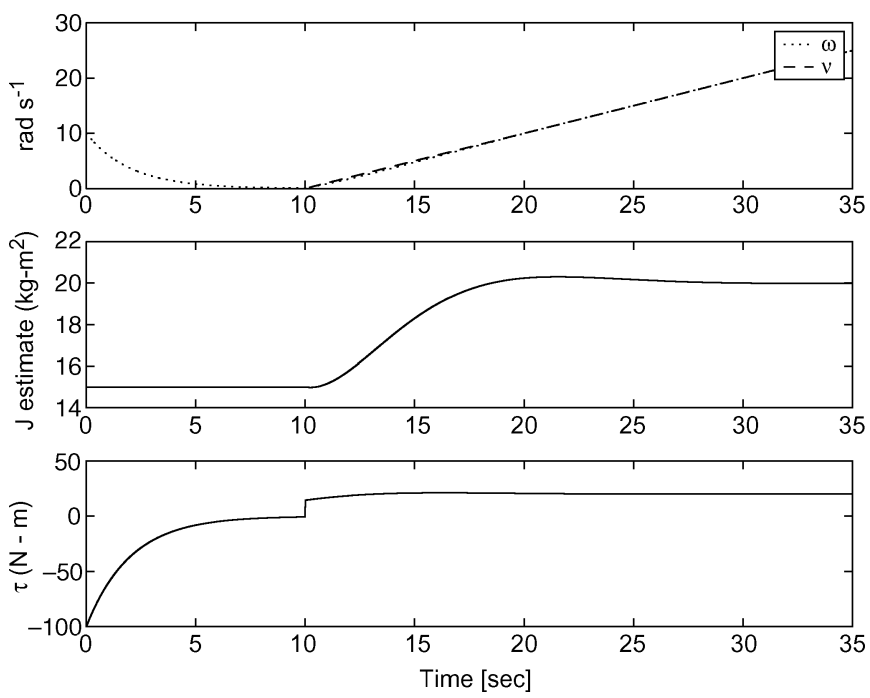

Fig. 2. Angular velocities, inertia estimate, and control torque for (14) in Example 2.

gains are the same as in the previous example, where as $\tilde{\omega}(0)$ is chosen to be 10 and $5 \mathrm{rad} / \mathrm{s}$, respectively. The angular velocity tracking error, inertia estimate error, and applied input torque are shown in Figs. 2 and 3. In both cases, the inertia estimates converge to the actual value.

\section{AdAPTIVE FEEDBACK LINEARIZATION CONTROL WITH NONAFFINE INPUT}

In Section II, we developed an adaptive controller that globally tracks angular velocity commands. We now consider the general case of an unknown nonaffine control input modeled by (2).

As mentioned in Section II, the single degree of freedom attitude dynamics with nonlinear actuation are modeled by

$$
\dot{\omega}=\frac{1}{J} h(u)
$$

where $\omega$ is the angular velocity, $u$ is the control signal, $J$ is the moment of inertia, and $h: \mathbb{R} \mapsto \mathbb{R}$ is an unknown input 

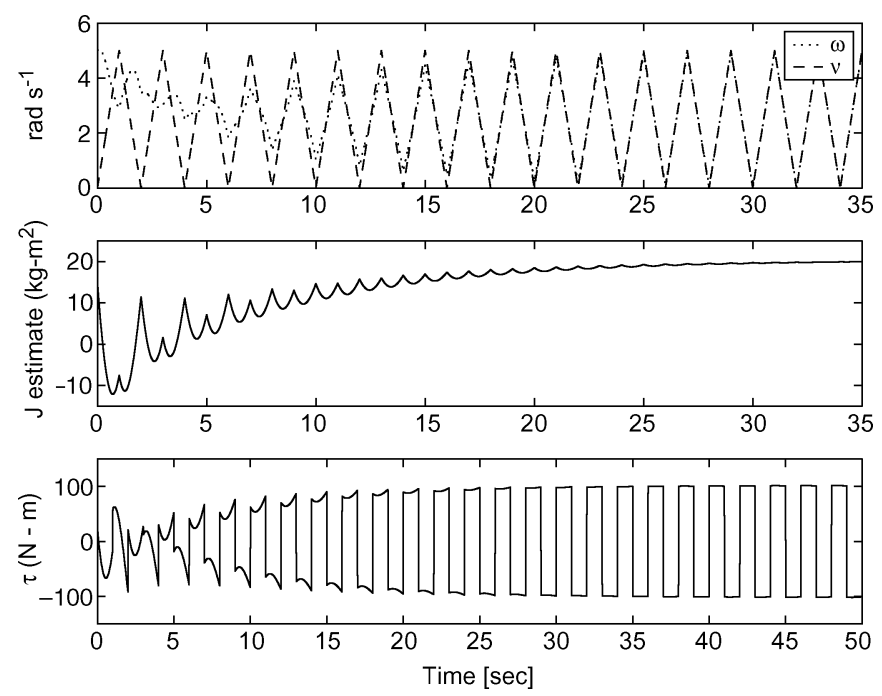

Fig. 3. Angular velocities, inertia estimate, and control torque for (15) in Example 2.

mapping that is continuous and onto, but not necessarily one-toone. Thus, $h$ may not have an inverse.

Assumption 1: The input nonlinearity $h(\cdot)$ is a polynomial.

Thus, the input nonlinearity can be written as

$$
h(u)=h_{l} u^{l}+h_{l-1} u^{l-1}+\ldots+h_{1} u+h_{0} .
$$

Assumption 2: The sign of the coefficient $h_{l}$ in (17) is known, and there exists a known $\beta>0$, such that $\left|h_{l}\right| \geq \beta$.

Remark 2: Assumption 2 does not require that the partial derivative $\partial h / \partial u$ of $h(u)$ be bounded away from zero or knowledge of its sign as in [15]-[18]. Indeed, $\partial h / \partial u$ is allowed to assume any real value.

Define $\hat{h}: \mathbb{R} \mapsto \mathbb{R}$ as $\hat{h}(u)=\lambda u$, where $\lambda \in \mathbb{R}$ satisfies $\operatorname{sign}(\lambda)=\operatorname{sign}\left(h_{l}\right)$. Then, we define an approximately linearizing feedback control law

$$
u=\hat{h}^{-1}(v)=\frac{v}{\lambda}
$$

where $v \triangleq \hat{h}(u)$. Next, we define the input model error $\Delta$ : $\mathbb{R} \mapsto \mathbb{R}$ by

$$
\Delta(u) \triangleq h(u)-\hat{h}(u)=h(u)-v
$$

so that $\Delta\left(\hat{h}^{-1}(v)\right)=h(v / \lambda)-v$ and $\tau=h(u)=\Delta(u)+v$. Then

$$
\dot{\omega}=\frac{1}{J} \tau=\frac{1}{J} h(u)=\frac{1}{J}\left[v+\Delta\left(\hat{h}^{-1}(v)\right)\right] .
$$

Now write $v=v_{a}-v_{c}$, so that

$$
\dot{\omega}=\frac{1}{J}\left[v_{a}+\Delta\left(\hat{h}^{-1}(v)\right)-v_{c}\right]
$$

where $v_{a}$ is the torque specified by the adaptive algorithm (4), (5) for the system (1), given by

$$
v_{a}=-k \tilde{\omega}+\dot{\nu}(t) \hat{J}
$$

and $v_{c}$ is the torque used to cancel the model error $\Delta\left(\hat{h}^{-1}(v)\right)$.
Next, it follows from Assumption 1 and Lemma 3 in Appendix $\mathrm{C}$ that $h(\cdot)$ is an odd degree polynomial and there exists $M \in \mathbb{R}^{l+1}$ such that, for all $v \in \mathbb{R}$

$$
\Delta\left(\hat{h}^{-1}(v)\right)=M^{\mathrm{T}} \sigma(v)
$$

where $\sigma(v) \triangleq\left[\begin{array}{lllll}v^{l} & v^{l-1} & \ldots & v & 1\end{array}\right]^{\mathrm{T}}$. Using (23), (21) can be written as

$$
\dot{\omega}=\frac{1}{J}\left[v_{a}+M^{\mathrm{T}} \sigma(v)-v_{c}\right] .
$$

To approximately cancel $M^{\mathrm{T}} \sigma(v)$ in (24), we use an estimate of $M^{\mathrm{T}} \sigma(v)$ given by

$$
v_{c}=\hat{M}^{\mathrm{T}} \sigma(v)
$$

where $\hat{M} \in \mathbb{R}^{l+1}$ is an estimate of $M$. Hence, it follows from (24) that

$$
\dot{\omega}=\frac{1}{J}\left[v_{a}+(M-\hat{M})^{\mathrm{T}} \sigma(v)\right]
$$

where $v$ is a solution of (25), which can be written as

$$
v+\hat{M}^{\mathrm{T}} \sigma(v)=v_{a}
$$

Denote $\hat{M} \in \mathbb{R}^{l+1}$ as

$$
\hat{M}=\left[\begin{array}{l}
\hat{m}_{0} \\
\hat{M}_{l}
\end{array}\right]
$$

where $\hat{m}_{0} \in \mathbb{R}$ and $\hat{M}_{l} \in \mathbb{R}^{l}$. Furthermore, define

$$
\bar{m}_{0} \triangleq \begin{cases}\frac{\beta}{|\lambda|}-1, & l=1 \\ \frac{\beta}{\left|\lambda^{l}\right|}, & l>1\end{cases}
$$

and initialize $\hat{m}_{0}(0) \in\left[\bar{m}_{0}, \infty\right)$ and $\hat{M}_{l}(0) \in \mathbb{R}^{l}$. The estimate $\hat{M}$ is then updated according to the adaptation law

$$
\begin{aligned}
& \dot{\hat{m}}_{0}= \begin{cases}g \tilde{\omega} v^{l}, & \text { if either } \hat{m}_{0}>\bar{m}_{0} \\
0, & \text { or if } \hat{m}_{0}=\bar{m}_{0} \text { and } \tilde{\omega} v^{l} \geq 0\end{cases} \\
& \dot{\hat{M}}_{l}=G \tilde{\omega} \sigma_{l}(v)
\end{aligned}
$$

where $G \in \mathbb{R}^{l \times l}$ is a positive-definite adaptation gain matrix, $g>0$, and $\sigma_{l}(v)=\left[\begin{array}{llll}v^{l-1} \ldots & \cdots & 1\end{array}\right]^{\mathrm{T}}$. Notice that (29) guarantees that $\left[\bar{m}_{0}, \infty\right)$ is an invariant set for $\hat{m}_{0}(t)$ and, hence, if $\hat{m}_{0}(0) \in\left[\bar{m}_{0}, \infty\right)$, then $\hat{m}_{0}(t) \geq \bar{m}_{0}$ for all time $t \geq 0$.

In summary, the control input $u$ is computed from (18), where $v$ is obtained from solving (27) with $v_{a}$ in (27), given by (22). The parameters $\hat{M}$ in (27) and $\hat{J}$ in (22) are solutions of (29), (30), and (4). In the next section, we see that the adaptive feedback linearization controller (18), globally tracks an arbitrary command signal.

\section{ANALySis OF THE AdAPTIVE FEEDBACK LINEARIZATION CONTROLLER}

In this section, we analyze the stability of the adaptive feedback linearization controller and prove global convergence of the angular velocity error $\tilde{\omega}$ to zero. Note that since (27) is implicit in $v$, we need to solve an algebraic equation in $v$ to compute the control input $u$. Hence, (27) must have at least one real 
solution. As shown in Lemma 4 in Appendix $\mathrm{C}$, the initialization for $\hat{m}_{0}(0)$ given by $\hat{m}_{0}(0) \in\left[\bar{m}_{0}, \infty\right)$ and $\hat{M}_{l}(0) \in \mathbb{R}^{l}$, where $\bar{m}_{0}$ is given by (28), guarantees existence of a solution to the algebraic (27).

However, since the solution $v$ of (27), and, hence, $u$ given by (18), might not be a continuous function of the states $\omega, \hat{J}$, and $\hat{M}$, the closed-loop vector field might not be continuous with respect to the states. Note that the closed-loop vector field can be discontinuous even if the input nonlinearity $h(u)$ has an inverse that is Lipschitz; the discontinuity is due to the fact that the approximation of the error $\Delta(\hat{h}(v))$ given by $\hat{M}^{\mathrm{T}} \sigma(v)$, may result in a solution $v$ of (27), and hence, a feedback control input $u$ in (18) that is not a continuous function of the states.

We, thus, require that the closed-loop system have at least one generalized solution in the sense of Filippov or Krasovskii [19], [20], [23], [24]. It may be noted that adaptive controllers developed in [17], [18], and [22] for nonaffine systems, implicitly make the assumption of existence of solutions for a system whose closed-loop vector field might not be Lipschitz or even continuous.

Consider the system (16), the control (18), and the adaptation law (4), (29), and (30), where $k>0, q>0, G$ is positive definite, and $g>0$. Defining the error $\tilde{M} \triangleq \hat{M}-M$, the closed-loop dynamics (4), (16), (22), (27), (29), and (30) can be written in error coordinates as

$$
\begin{aligned}
& \dot{\tilde{\omega}}=\frac{1}{J}\left[-k \tilde{\omega}+\dot{\nu} \tilde{J}-\tilde{M}^{T} \sigma(v)\right] \\
& \dot{\tilde{J}}=-q \dot{\nu} \tilde{\omega} \\
& \dot{\tilde{m}}_{0}= \begin{cases}g \tilde{\omega} v^{l}, & \text { if either } \hat{m}_{0}>\bar{m}_{0} \\
& \text { or if } \hat{m}_{0}=\bar{m}_{0} \text { and } \tilde{\omega} v^{l} \geq 0 \\
0, & \text { else }\end{cases} \\
& \dot{\tilde{M}}_{l}=G \tilde{\omega} \sigma_{l}(v)
\end{aligned}
$$

where

$$
v+(\tilde{M}+M)^{\mathrm{T}} \sigma(v)=-k \tilde{\omega}+\dot{\nu}(\tilde{J}+J) .
$$

Next, we prove global convergence of the angular velocity error $\tilde{\omega}$ to zero for the closed-loop system (31)-(35).

Theorem 3: Assume that $\nu$ is $C^{1}$ and $\dot{\nu}$ is bounded on $[0, \infty)$. Consider the closed-loop system (4), (16), (18), (29), and (30), where $k>0, q>0, G$ is positive definite, and $g>0$, written in the error coordinates (31)-(35). Assume that (31)-(34) has a local generalized solution for all $\tilde{\omega}(0), \tilde{J}(0)$, $\tilde{M}_{l}(0), \hat{m}_{0}(0) \in\left[\bar{m}_{0}, \infty\right)$, and $v \in \mathbb{R}$, where $\bar{m}_{0}$ is given by (28). Choose $\hat{m}_{0}(0) \in\left[\bar{m}_{0}, \infty\right)$. Then, the closed-loop dynamics of (31)-(35) have a generalized solution that satisfies $\hat{m}_{0}(t) \in\left[\bar{m}_{0}, \infty\right)$ for all $t \geq 0$, and the zero equilibrium solution of (31)-(35) is Lyapunov stable. Furthermore, $\lim _{t \rightarrow \infty} \tilde{\omega}(t)=0, \lim _{t \rightarrow \infty} \tilde{\tilde{J}}(t)=0$, and $\lim _{t \rightarrow \infty} \dot{\tilde{M}}(t)=0$ for all $\tilde{\omega}(0)$, $\tilde{J}(0)$, and $\tilde{M}_{l}(0)$.

Proof: Lemma 5 in Appendix C implies that there exists a generalized solution of (31)-(35) over some maximal interval $[0, T)$ that satisfies the constraint $\hat{m}_{0}(t) \in\left[\bar{m}_{0}, \infty\right)$. We shall next show that this solution is contained in a compact set for all $t \in[0, T)$. Then by standard continuation arguments, it is clear that $T$ is arbitrarily large and, hence, this generalized solution exists for all $t \geq 0$. Then clearly, this generalized solution also satisfies the constraint $\hat{m}_{0}(t) \in\left[\bar{m}_{0}, \infty\right)$ for $t \in[0, \infty)$.

We next prove that a solution of (31)-(35) lies in a compact set over the maximal interval of existence $[0, T)$. Consider the radially unbounded, positive-definite Lyapunov candidate

$$
V(\tilde{\omega}, \tilde{J}, \tilde{M})=\frac{J}{2} \tilde{\omega}^{2}+\frac{1}{2 q} \tilde{J}^{2}+\frac{1}{2} \tilde{M}_{l}^{\mathrm{T}} G^{-1} \tilde{M}_{l}+\frac{1}{2 g} \tilde{m}_{0}^{2}
$$

Then, along a trajectory of the system

$$
\dot{V}(\tilde{\omega}, \tilde{J}, \tilde{M})=J \tilde{\omega} \dot{\tilde{\omega}}+\frac{1}{q} \tilde{J} \dot{\tilde{J}}+\tilde{M}_{l}^{\mathrm{T}} G^{-1} \dot{\tilde{M}}_{l}+\frac{1}{g} \tilde{m}_{0} \dot{\tilde{m}}_{0}
$$

Substituting (31)-(35) into (37) yields

$$
\dot{V}(\tilde{\omega}, \tilde{J}, \tilde{M})= \begin{cases}-k \tilde{\omega}^{2}, & \text { if either } \hat{m}_{0}>\bar{m}_{0} \\ -k \tilde{\omega}^{2}-\tilde{m}_{0} \tilde{\omega} v^{l}, & \text { or if } \hat{m}_{0}=\bar{m}_{0} \text { and } \tilde{\omega} v^{l} \geq 0 ;\end{cases}
$$

In the first case $\dot{V}=-k \tilde{\omega}^{2} \leq 0$. In the second case, $\dot{V}=$ $-k \tilde{\omega}^{2}-\tilde{m}_{0} \tilde{\omega} v^{l}$. We now show that in the second case, $\tilde{m}_{0} \tilde{\omega} v^{l} \geq$ 0 and hence, $\dot{V} \leq-k \tilde{\omega}^{2} \leq 0$ for both cases.

Consider the second case. Since the first case holds for $\hat{m}_{0}>$ $\bar{m}_{0}$, it follows that $\hat{m}_{0}=\bar{m}_{0}$ for the second case, since $\hat{m}_{0}(t) \geq$ $\bar{m}_{0}$ for all $t \in[0, T)$ from Lemma 5 in Appendix C. Therefore, $\tilde{m}_{0}=\hat{m}_{0}-m_{0}=\bar{m}_{0}-m_{0}$. However, from Lemma 6 in Appendix $\mathrm{C}$, we know that $\bar{m}_{0}-m_{0} \leq 0$, and hence, $\tilde{m}_{0} \leq 0$. Next, if the second case holds true then $\tilde{\omega} v^{l}<0$ since the negation, namely, $\tilde{\omega} v^{l} \geq 0$, implies the first case. Thus, $\tilde{m}_{0} \tilde{\omega} v^{l} \geq 0$, and hence, $\dot{V}(\tilde{\omega}, \tilde{J}, \tilde{M}) \leq 0$, which shows that $\dot{V}$ is negative semidefinite. Thus, the solution is contained in the compact set

$$
\begin{aligned}
& \mathcal{K}=\left\{(\tilde{\omega}, \tilde{J}, \tilde{M}) \in \mathbb{R}^{2} \times\left[\bar{m}_{0}, \infty\right)\right. \times \mathbb{R}^{l}: V(\tilde{\omega}, \tilde{J}, \tilde{M}) \\
&\leq V(\tilde{\omega}(0), \tilde{J}(0), \tilde{M}(0))\} .
\end{aligned}
$$

Hence, the solution exists for all $t \geq 0$ and satisfies $\hat{m}_{0}(t) \geq$ $\bar{m}_{0}$ for $t \in[0, \infty)$. Clearly, the zero equilibrium solution of (31)-(35) is Lyapunov stable.

Next, [[21], Th. 8.4] implies that, for all initial conditions $\tilde{\omega}(0), \tilde{J}(0), \tilde{M}_{l}(0)$ and $\hat{m}_{0}(0) \geq \bar{m}_{0}$, the solutions of $(31)-(35)$ are bounded and approach the set $E \triangleq \dot{V}^{-1}(0)=\{[\tilde{\omega} \tilde{J} \tilde{M}] \in$ $\left.\mathbb{R}^{2} \times\left[\bar{m}_{0}, \infty\right) \times \mathbb{R}^{l}: \tilde{\omega}=0\right\}$. Hence, $\tilde{\omega}(t) \rightarrow 0$ as $t \rightarrow \infty$. Since $\tilde{\omega}(t) \rightarrow 0$ as $t \rightarrow \infty$ and $\dot{\nu}$ is bounded, it follows from (32) and (33) that $\dot{\tilde{J}}(t) \rightarrow 0$ and $\dot{\tilde{M}}(t) \rightarrow 0$ as $t \rightarrow \infty$. Thus, $\dot{\hat{J}}(t) \rightarrow 0$ and $\dot{\hat{M}}(t) \rightarrow 0$ as $t \rightarrow \infty$.

Since the vector field of (31)-(35) is not necessarily Lipschitzian, the uniqueness of the solution cannot be guaranteed.

Remark 3: Note that [[21], Th. 8.4] requires that the vector field of the closed-loop be locally Lipschitz and continuous for all $t \geq 0$, which is not true for the closed-loop system given by (31)-(35). However, the requirement of a continuous and locally Lipschitz vector field can be weakened to the vector field being bounded over every compact subset of the state space. In the case of the closed-loop system given by (31)-(35), since $\nu$ is $C^{1}$, the image of the function given by the vector field of the closed-loop (31)-(35) is bounded over every compact subset of $\mathbb{R}^{2} \times\left[\bar{m}_{0}, \infty\right) \times \mathbb{R}^{l}$. Hence, [[21], Th. 8.4] is applicable to the system given by (31)-(35). 


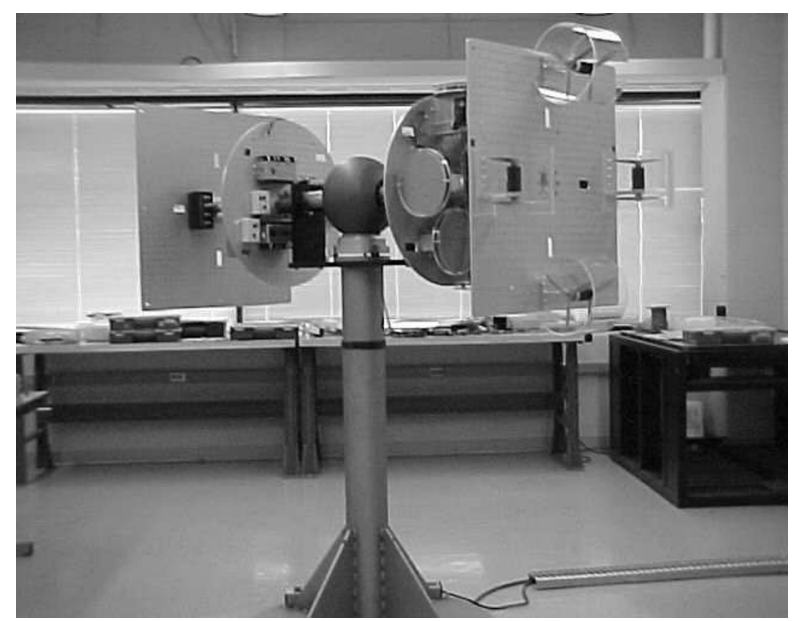

Fig. 4. Triaxial air bearing testbed. This testbed, which is based on a spherical air bearing, allows low friction, 3-D motion with unrestricted roll and yaw, and $\pm 45^{\circ}$ pitch.

\section{Triaxial Attitude Control Testbed}

\section{A. Mechanical Setup}

The experimental testbed (see Fig. 4) is based on a spherical air bearing manufactured by Space Electronics, Inc., Berlin, CT. An aluminum sphere of 11-in diameter floats on a thin film of air that exits from holes located in the surface of the cup. Air at 70 psi is supplied to the cup by means of a hose that passes through the center of the vertical support.

A one-piece 32-in stainless steel shaft passes through the center of the sphere and extends between a pair of 24-in circular mounting plates. This shaft is designed to withstand stresses that might otherwise distort the sphere. All mounting plates are made from 1/4-in aluminum alloy with 1/4-20 holes tapped in a 1-in grid. The 14-in aluminum extension shafts connect the circular mounting plates to the 30 -in $\times 30$-in square mounting plates. The distance between the square plates is $5 \mathrm{ft}$. All shafts have hollow interior to allow wiring through the sphere and between any two points. The total weight of the levitated components described thus far is $180 \mathrm{lb}$. At 70 psi air pressure, the air bearing can support an additional $180 \mathrm{lb}$ of components.

The spherical air bearing allows unrestricted motion in yaw (rotation about the vertical axis) and roll (rotation about the longitudinal shaft axis). The plates and shafts are designed to allow $\pm 45^{\circ}$ pitch (rotation about a horizontal axis) at all roll and yaw angles.

Once the main components are mounted, additional masses can be added to modify the final mass distribution. For planar rotation experiments, the center of mass is located along the vertical line that passes through the rotational center. This mass distribution balances pitch motion. However, when the center of mass is not located at the rotational center, the body possesses pendulum dynamics in roll, and thus, yields predominantly yaw dynamics for one-dimensional (1-D) experiments. For 3-D experiments, the center of mass can be located at the rotational center to balance the system in both roll and pitch.

\section{B. Control Hardware}

The Triaxial Attitude Control Testbed uses onboard sensors in the attitude control experiments. A three-axis magnetometer determines the direction of the Earth's magnetic north; a three-axis accelerometer measures gravitational and centripetal acceleration; and a three-axis gyro measures angular velocity. Only the gyros are needed for this paper.

The three-axis gyro is comprised of three Gyrochip Horizon rate sensors manufactured by Systron Donner, Concord, CA. The input range of these sensors is $\pm 90^{\circ} / \mathrm{s}$ and, according to specifications, their bandwidth is greater than $18 \mathrm{~Hz}$. Under static conditions, that is, $\omega=0$, we measured the rms gyro noise to be about $1.3 \mathrm{mV}$, which corresponds to $0.06^{\circ} / \mathrm{s}$. Since the gyro measurement range is $0-5 \mathrm{~V}$, the sensor dynamic range is found to be $71.7 \mathrm{~dB}$, or 12 analog-digital conversion bits. Operation of fan thrusters does not affect the gyro noise significantly.

We use an embedded processor developed by Quanser Consulting for realtime onboard processing. This processor is based on a 586 processor with $256 \mathrm{MB}$ RAM, 4 GB solid state hard disk, and Multi-Q I/O boards allowing up to 24 A/D channels, 24 D/A channels, and 16 encoder channels. The A/D and D/A channels have a resolution of 13 bits over a $\pm 5 \mathrm{~V}$ range. The A/D sampling occurs sequentially with an acquisition time of $20 \mu \mathrm{s} /$ channel, while the D/A conversion also occurs sequentially with a latency of $5 \mu \mathrm{s} / \mathrm{channel}$. The operating system is based on the Quanser Consulting WinCon realtime controller, which is compatible with the MathWorks RealTime Workshop for implementing controllers programmed in Simulink. Communication with the host PC for experiment monitoring, parameter modification, and data acquisition is accomplished through a wireless ethernet connection.

The Triaxial Attitude Control Testbed uses propeller thrusters for control actuation. The experiments described here use four propeller thrusters. These thrusters are based on Maxon motors and Copley amplifiers. Without the encoders mounted, these motors have a dual protruding shaft to which a pair of propellers is mounted to obtain direction-symmetric thrust. The Copley amplifiers for the thrusters are operated in velocity mode to provide a commandable torque.

\section{EXPERIMENTAL RESULTS}

\section{A. Preliminary Analysis}

In this and the subsequent section, we present experimental results performed on the Triaxial Attitude Control Testbed for the following cases:

1) simple proportional controller with no adaptation to the inertia or to the input nonlinearity;

2) adaptive controller designed, as in Section II, that adapts only to the unknown inertia but does not compensate for the input nonlinearity;

3) adaptive controller, as in Section II, that adapts only to the unknown inertia and does compensate for the input nonlinearity, using a piecewise linear approximation of the inverse of the input nonlinearity; and 
4) adaptive feedback linearization controller, as in Section III, that provides adaptation for the unknown inertia and for the unknown input nonlinearity.

As already mentioned, we consider only yaw motion of the testbed with two thrusters for actuation and one gyro for yaw-rate sensing. Experimental results pertaining to cases (1), (2), and (3) are presented in this section, while case (4) is considered in the next section.

To relate physical signals to measurements, let

$$
\omega=K_{\mathrm{gyro}} V_{\mathrm{gyro}}, \quad \tau=K_{\mathrm{fan}} V_{\mathrm{fan}}
$$

where $V_{\text {gyro }}$ is the voltage output of the gyro, $K_{\text {gyro }}$ is the conversion coefficient from $V_{\text {gyro }}$ in volts to $\omega$ in $\% / \mathrm{s}, V_{\text {fan }}$ is the voltage input to the thruster amplifiers, and $K_{\mathrm{fan}}$ is the conversion coefficient from $V_{\text {fan }}$ in volts to the control torque $\tau$ in newton meters. From (1), we see that $\dot{V}_{\text {gyro }}=V_{\text {fan }} / J^{\prime}$, where the scaled inertia $J^{\prime} \triangleq J K_{\text {gyro }} / K_{\text {fan. }}$. Note that the units of $J^{\prime}$ are seconds. We define $V_{\text {ref }} \triangleq \nu / K_{\text {gyro }}$ and $\tilde{V} \triangleq V_{\text {gyro }}-V_{\text {ref }}$. We can, thus, rewrite (4) as

$$
V_{\text {fan }}=-k^{\prime} \tilde{V}+\dot{V}_{\text {ref }} \hat{J}^{\prime}
$$

where $k^{\prime} \triangleq k K_{\text {gyro }} K_{\text {fan }}$ and $\hat{J}^{\prime}$ is the estimate of $J^{\prime}$. The adaptive law (5) can be written as

$$
\dot{\hat{J}}^{\prime}=-q^{\prime} \dot{V}_{\text {ref }} \tilde{V}
$$

where $q^{\prime} \triangleq q K_{\text {gyro }}^{3} / K_{\text {fan }}$. Comparing (4) and (5) with (39) and (40), it follows that the conversion coefficients are incorporated within the constants $q^{\prime}$ and $k^{\prime}$. It can be seen that $k^{\prime}$ is dimensionless and $q^{\prime}$ has units of $\mathrm{V} / \mathrm{s}^{2}$. Hence, we can apply the adaptive control algorithm of Section II without further calibration. However, to relate our results to physical motion, we calibrated the gyro voltage and found $K_{\text {gyro }}=45.5^{\circ} /(\mathrm{V} \cdot \mathrm{s})$. For the remainder of this section, we view $V_{\text {fan }}$ as the control signal.

\section{B. Experiments Using Proportional Controller and Using Adaptive Controller Without Compensation for Input Nonlinearity}

When $\nu$ is constant, the adaptive controller specializes to the proportional controller

$$
V_{\text {fan }}=-k^{\prime} \tilde{V} .
$$

Since the plant (1) is an integrator, the closed-loop system with the proportional controller (41) would yield zero steady-state error for step commands if the control input were affine.

The angular velocity $\omega(t)$ for the sinusoidal command $\nu(t)=$ $10 \sin .2 t^{\circ} / \mathrm{s}$ and a proportional gain of $k^{\prime}=20 \mathrm{~s} / \mathrm{V}^{2}$ is shown in Fig. 5. The angular velocity $\omega(t)$ converges to a periodic signal with rms value of about $2.6^{\circ} / \mathrm{s}$.

Now we use the second controller identified in Section VI-A for the same command. Fig. 6 shows $\omega(t)$ and $\nu(t)$ for $k^{\prime}=3$, $q^{\prime}=1000 \mathrm{~s} / \mathrm{V}^{2}$, and initial scaled inertia estimate $\hat{J}^{\prime}(0)=0 \mathrm{~s}$. The angular velocity error $\tilde{\omega}(t)$ shown in Fig. 7 converges to a

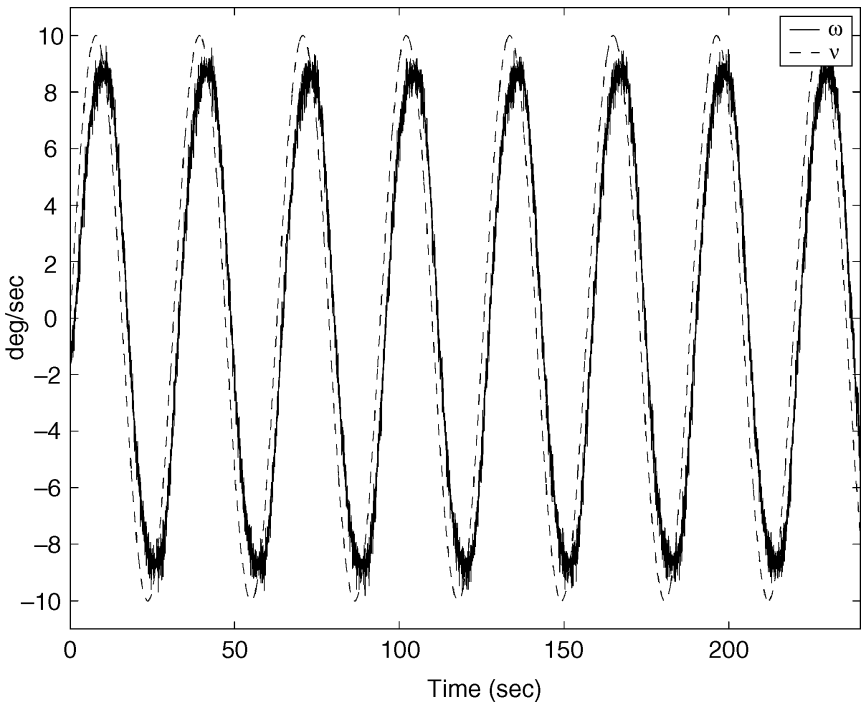

Fig. 5. Angular velocities $\omega(t)$ and $\nu(t)$ using proportional controller for $\nu(t)=10 \sin .2 t \% \mathrm{~s}$.

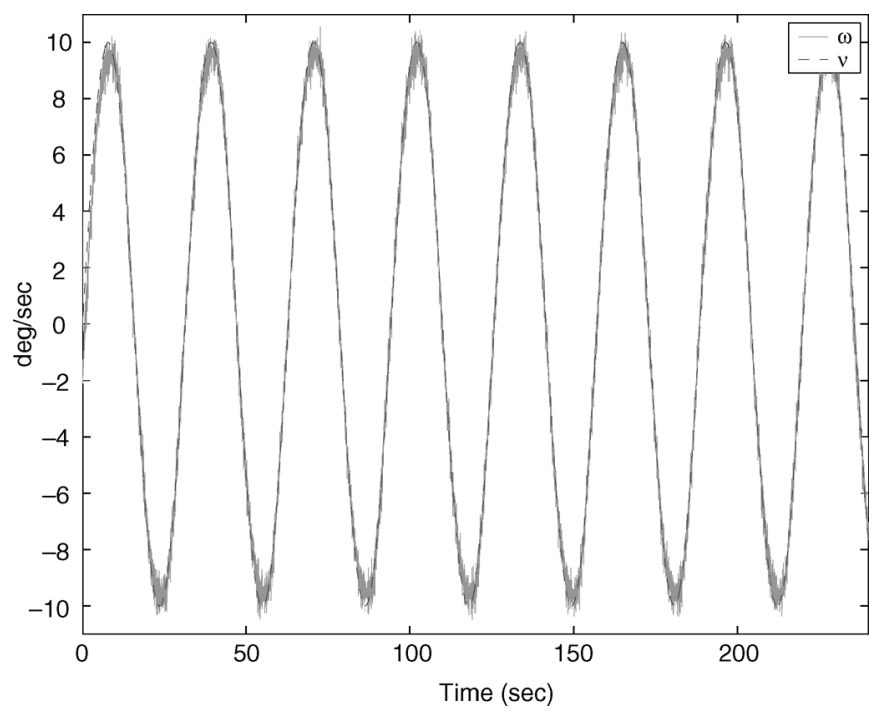

Fig. 6. Angular velocities $\omega(t)$ and $\nu(t)$ using the adaptive controller for $\nu=$ $10 \sin .2 t^{\circ} / \mathrm{s}$.

periodic signal with rms value of about $0.25 \%$ and mean value of $0.0061 \%$ s. Fig. 8 gives the scaled inertia estimates obtained with $\hat{J}^{\prime}(0)=100 \mathrm{~s}$ and $\hat{J}^{\prime}(0)=0 \mathrm{~s}$. The scaled inertia estimate converges to a periodic signal with mean value of about $37.9 \mathrm{~s}$ and a peak-to-peak amplitude of about $4.5 \mathrm{~s}$. Simulations and experiments (not shown) indicate that the value of the inertia estimate varies with the frequency of the command signal as a consequence of the nonaffine control input.

\section{Experiments Using Adaptive Controller With Approximate Compensation of Input Nonlinearity}

To determine whether the oscillation of $\hat{J}^{\prime}$ in Fig. 8 is due to the nonaffine control input, we plot (see Fig. 9) $\dot{V}_{\text {gyro }}$ in volts per second (obtained by numerically differentiating the measured $V_{\text {gyro }}$ ) versus $V_{\text {fan }}$ in volts as computed by the adaptive 


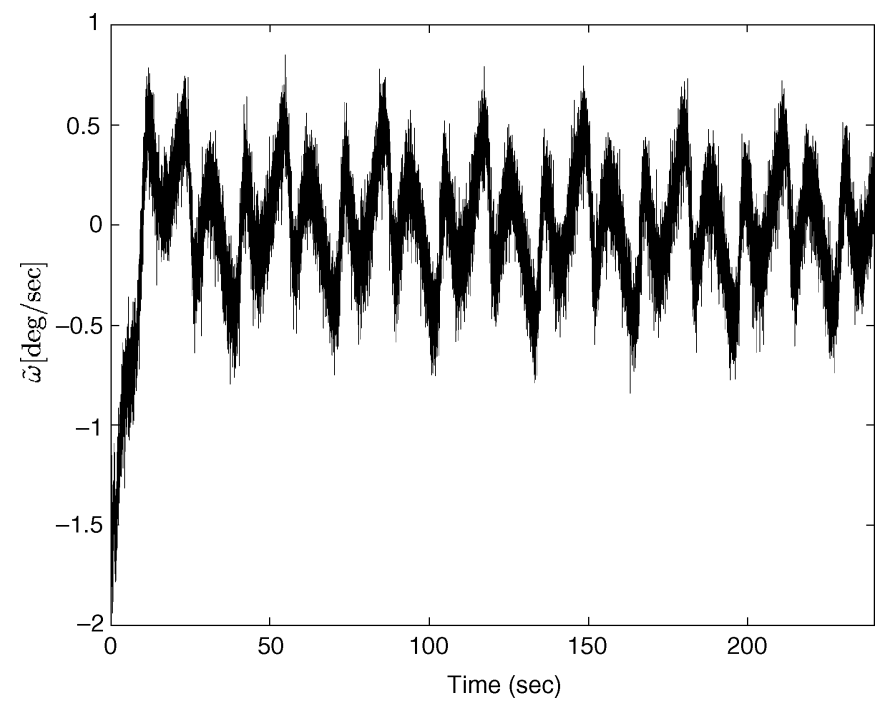

Fig. 7. Angular velocity tracking error using the adaptive controller for $\nu(t)=$ $10 \sin .2 t^{\circ} / \mathrm{s}$.

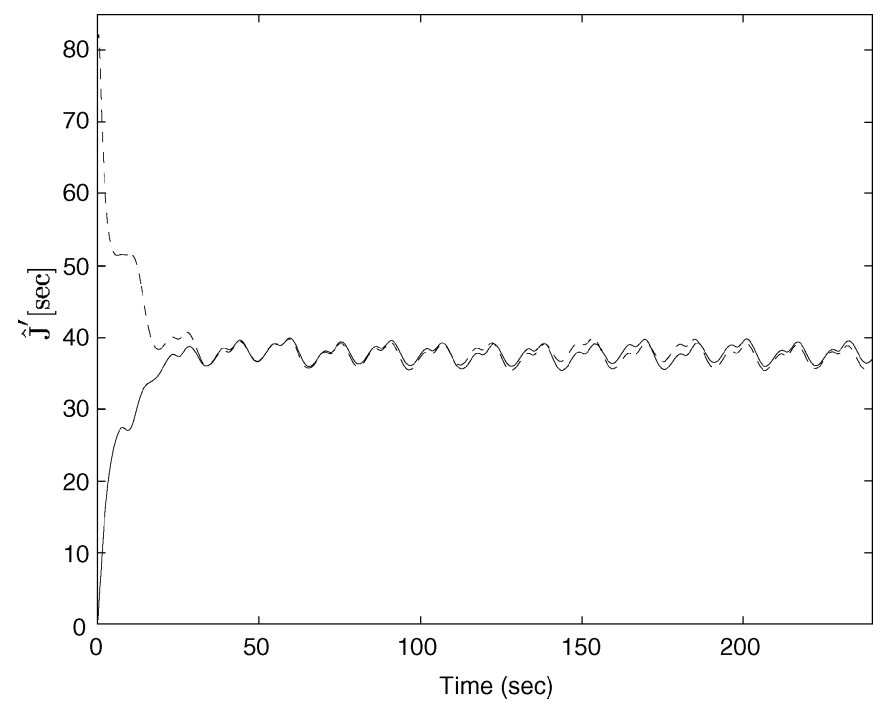

Fig. 8. Scaled inertia estimate using the adaptive controller for $\nu(t)=$ $10 \sin .2 t^{\circ} / \mathrm{s}, \hat{J}(0)=0 \mathrm{~s}$, and $\hat{J}(0)=100 \mathrm{~s}$.

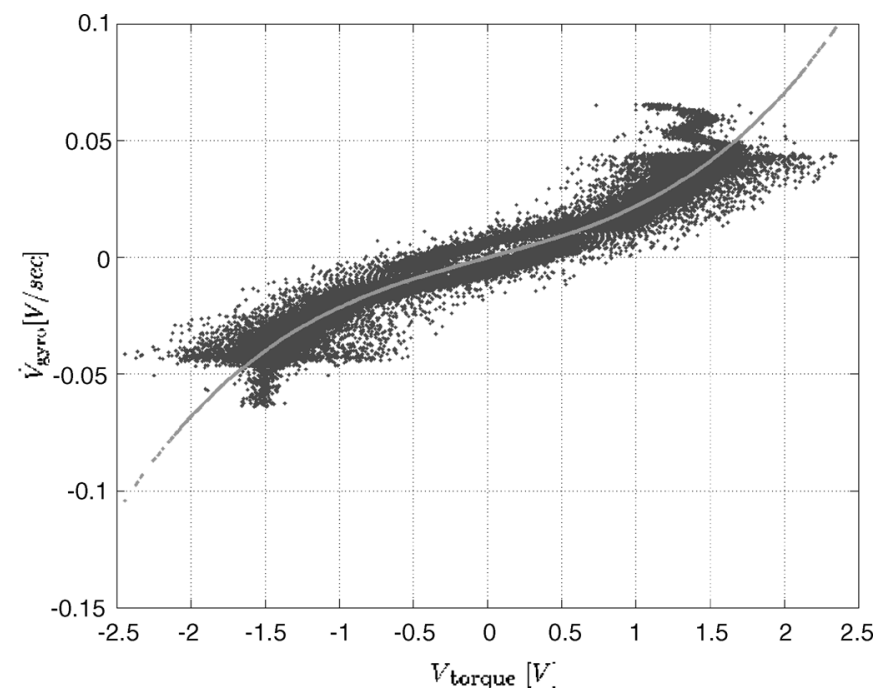

Fig. 9. $\quad \dot{V}_{\text {gyro }}$ versus $V_{\text {fan }}$ for $\nu(t)=10 \sin .1 t^{\circ} / \mathrm{s}$.

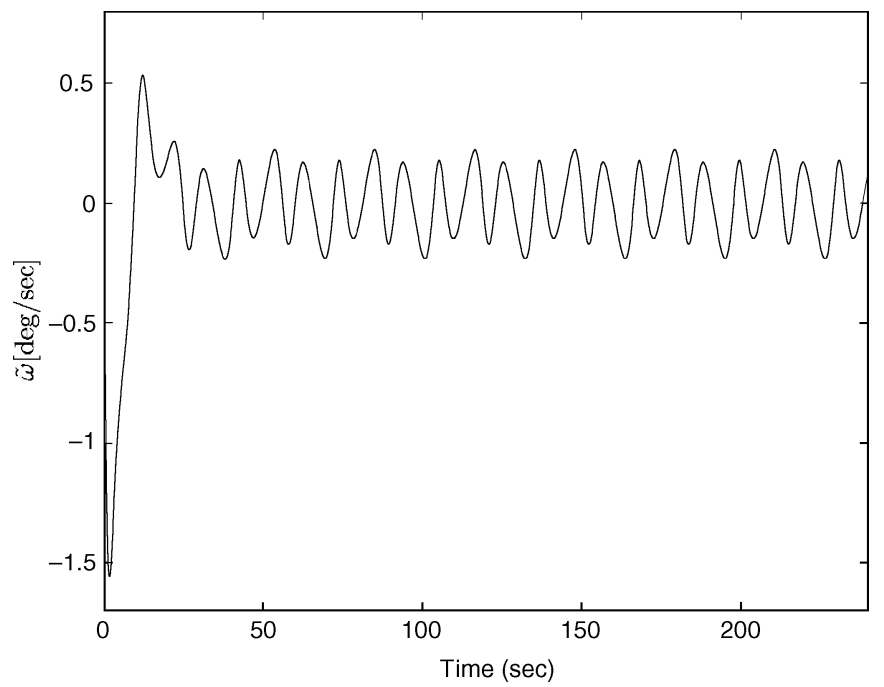

Fig. 10. Simulated angular velocity error with actuator nonlinearity.

algorithm during an experiment. Fig. 9 shows that $\dot{V}_{\text {gyro }}$ is a nonlinear function of the computed moment $V_{\mathrm{fan}}$, that is

$$
\dot{V}_{\text {gyro }}=\mathcal{N}\left(V_{\text {fan }}\right) \text {. }
$$

The input nonlinearity observed in the actuators is largely due to the aerodynamics of the propeller which generates the required thrust. The data are fit by the cubic polynomial

$$
\mathcal{N}(x)=0.1677 x^{3}+0.0117 x^{2}+0.6747 x+0.0078
$$

as shown in Fig. 9. From (5), (38), and (42), we have

$$
\dot{\omega}=K_{\text {gyro }} \mathcal{N}\left(\frac{-k \tilde{\omega}+\dot{\nu} \hat{J}}{K_{\text {fan }}}\right) .
$$

Hence, from (1) the torque input is given by

$$
\tau=J K_{\text {gyro }} \mathcal{N}\left(\frac{-k \tilde{\omega}+\dot{\nu} \hat{J}}{K_{\text {fan }}}\right) .
$$

To check whether the nonlinearity (43) could cause the residual oscillations in Fig. 8, the cubic nonlinearity (43) is included in a simulation of the adaptive closed-loop system for the command $\nu(t)=10 \sin .2 t^{\circ} / \mathrm{s}$. Figs. 10 and 11 suggest that this nonlinearity could indeed cause oscillations similar to those observed from the testbed. The angular velocity error $\tilde{\omega}$, shown in Fig. 10, converges to a periodic signal with rms value of about $0.14 \% \mathrm{~s}$ and mean value of $0.0066^{\circ} / \mathrm{s}$. The scaled inertia estimate converges to a periodic signal with value of $39.15 \mathrm{~s}$ and a peak-to-peak amplitude of about $3 \mathrm{~s}$.

For the periodic command signal $\nu(t)=10 \sin .2 t^{\circ} / \mathrm{s}$, Figs. 10 and 11 show the resulting $\tilde{\omega}(t)$ and $\hat{J}(t)$. We obtain a piecewise linear approximation of the cubic nonlinearity, and invert this piecewise linear function. The cubic nonlinearity and the inverse of the piecewise linear approximation are shown in Fig. 12.

The simulated response of the closed-loop system (see Figs. 13 and 14) indicates that the piecewise linear inverse approximately linearizes the nonlinearity and reduces oscillations in the angular velocity error and scaled inertia estimate. The rms value of $\tilde{\omega}$ is about $0.02^{\circ} / \mathrm{s}$, and the mean value is $0.01^{\circ} / \mathrm{s}$, 


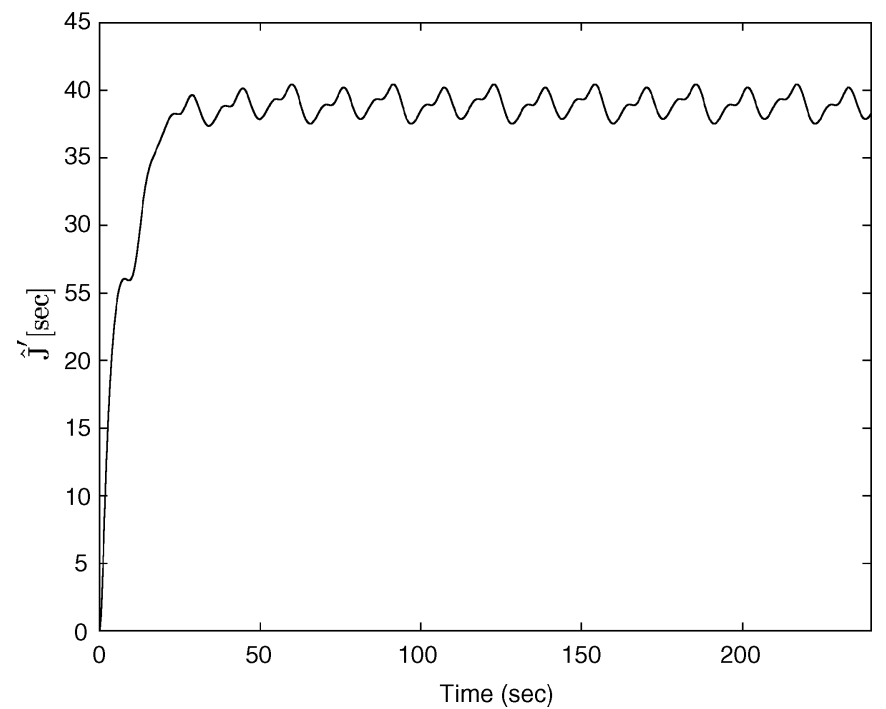

Fig. 11. Simulated scaled inertia estimate with actuator nonlinearity.

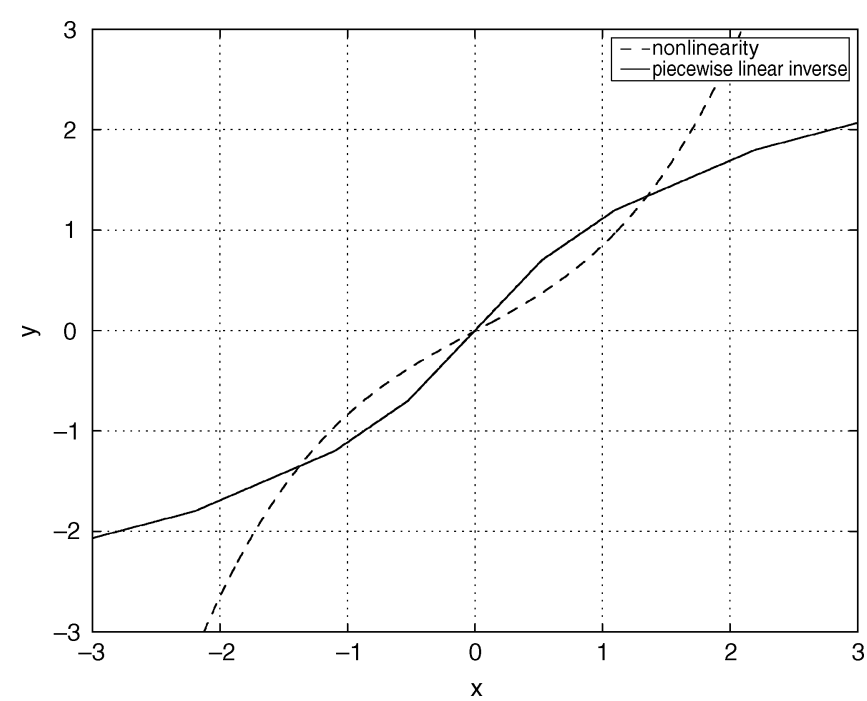

Fig. 12. Cubic actuator nonlinearity (43) and an approximate piecewise linear inverse.

which is below the noise level of the gyro. The mean value of the scaled inertia estimate is about $40.5 \mathrm{~s}$ with a peak-to-peak amplitude of about $0.9 \mathrm{~s}$. Furthermore, simulations (not shown) indicate that the scaled inertia estimates converge to the same value for different values of the frequency of $\nu(t)$.

The inverted actuator nonlinearity with the adaptive controller is implemented on the triaxial testbed and the results are shown in Figs. $15-17$. The rms value of $\tilde{\omega}$ is about $0.15^{\circ} / \mathrm{s}$ and the mean value is $0.011 \%$ s. The mean value of the scaled inertia is about $39.6 \mathrm{~s}$ and the peak-to-peak amplitude of oscillation is about $2.5 \mathrm{~s}$. Although oscillations in the angular velocity error and inertia estimates are reduced, they are not entirely eliminated. Sensor noise may account for some part of the oscillations in Fig. 15.

\section{Inertia of the Triaxial Testbed}

To determine the actual inertia in $\mathrm{kg} \cdot \mathrm{m}^{2}$, test masses are added at known distances from the rotational axis. Let $\Delta J, J^{\prime}$, and $J^{\prime \prime}$ denote the change in inertia, the scaled inertia, and the

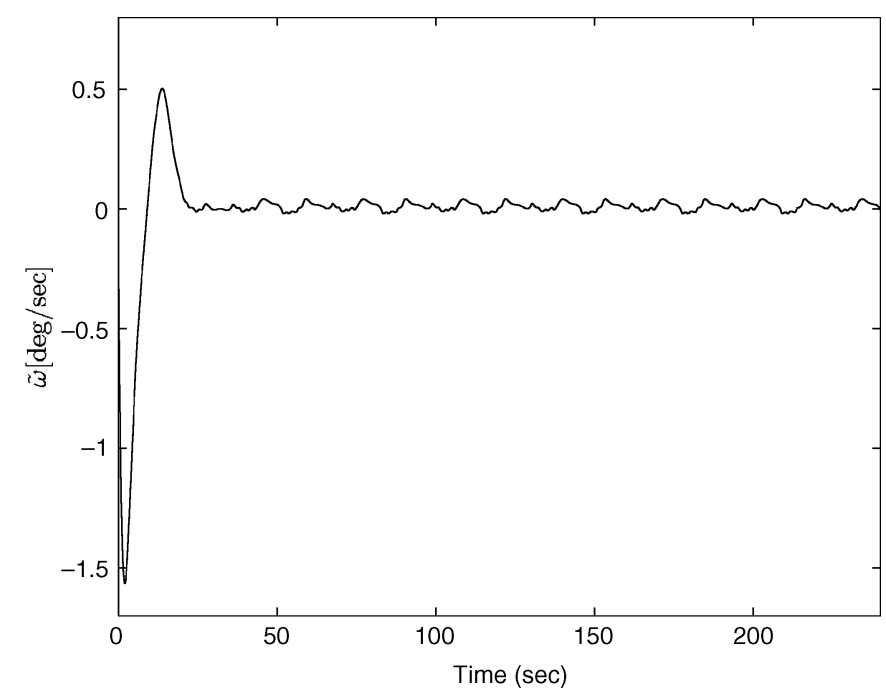

Fig. 13. Simulated angular velocity error with inverted actuator nonlinearity.

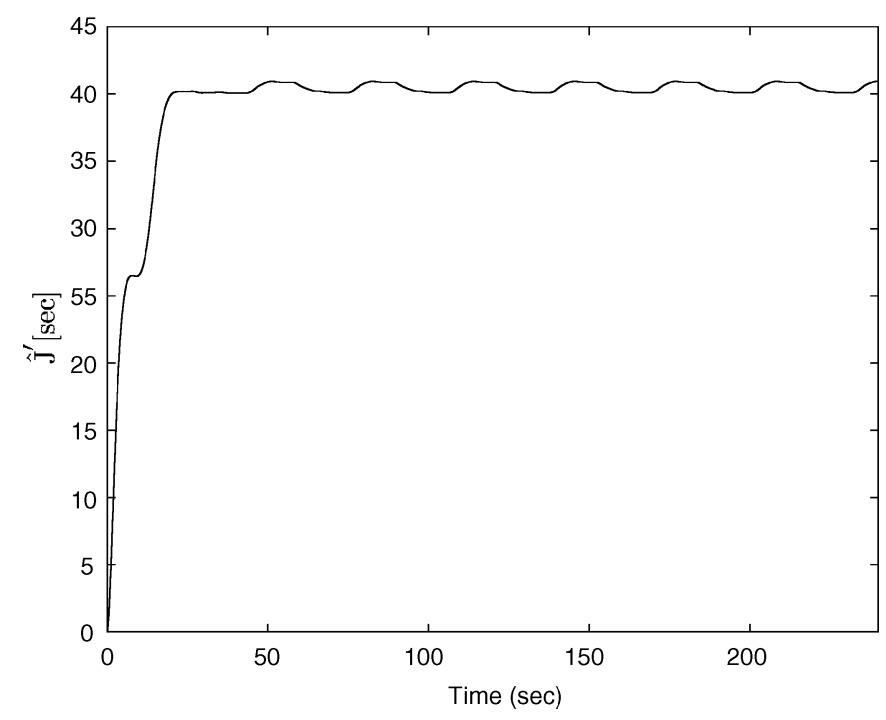

Fig. 14. Simulated scaled inertia estimate with inverted actuator nonlinearity.

scaled inertia from an experiment with added test masses, respectively. Hence

$$
J^{\prime \prime}=(J+\Delta J) \frac{K_{\text {gyro }}}{K_{\text {fan }}}
$$

and the inertia in $\mathrm{kg} \cdot \mathrm{m}^{2}$ is given by

$$
J=\frac{-J^{\prime} \Delta J}{J^{\prime}-J^{\prime \prime}} \text {. }
$$

A total mass of $5.11 \mathrm{~kg}$ is added to the two square mounting plates of the testbed, each at a distance of $0.75 \mathrm{~m}$ from the rotational axis. Hence, $\Delta J=2.87 \mathrm{~kg} \cdot \mathrm{m}^{2}$. Since the scaled inertia estimate $\hat{J}^{\prime \prime}$ is about $39.6 \mathrm{~s}$, it follows from (47) that the actual moment of inertia is $J=66.6 \mathrm{~kg} \cdot \mathrm{m}^{2}$.

\section{EXPERIMENTS USING ADAPTIVE FEEDBACK LINEARIZING CONTROLLER}

In this section, we discuss implementation issues concerning the adaptive feedback linearization controller and compare the simulation with experimental results. These results are also 


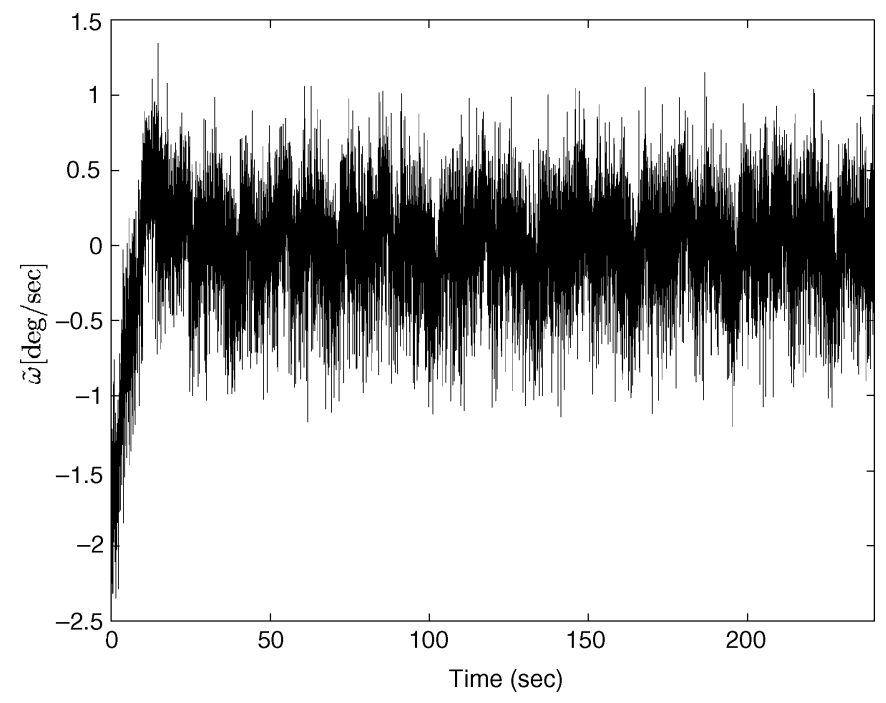

Fig. 15. Experimental angular velocity error with inverted actuator nonlinearity.

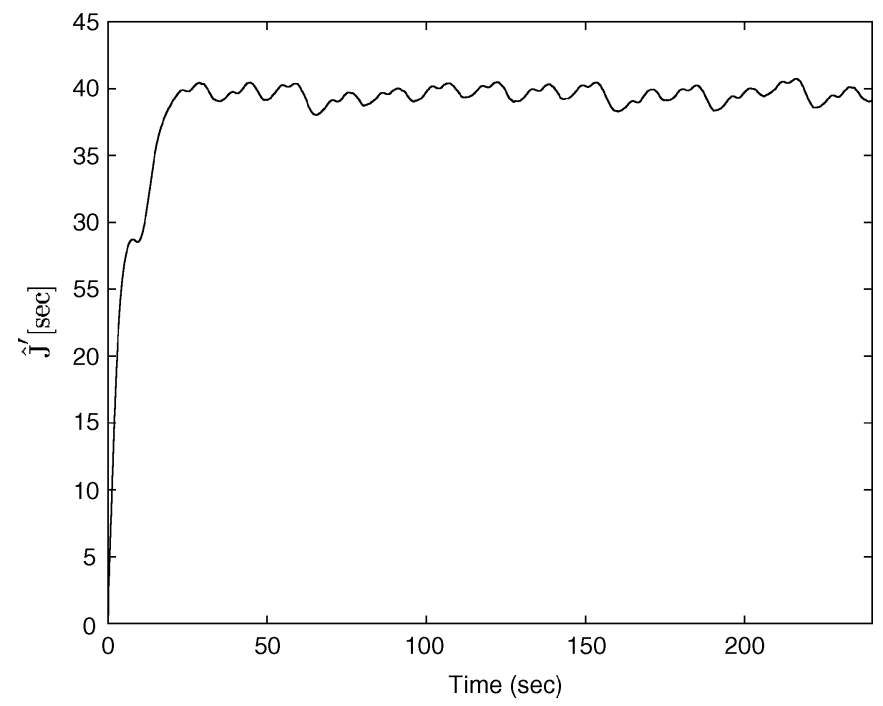

Fig. 16. Experimental scaled inertia estimate with inverted actuator nonlinearity.

compared with the results obtained for the three cases presented in Section VI. As discussed in Section IV, the adaptive feedback linearization controller involves the algebraic (27) which is guaranteed by Lemma 1 to have at least one real solution for all $v_{a} \in \mathbb{R}, \hat{M}_{l} \in \mathbb{R}^{l}$ and $\hat{m}_{0} \in\left[\bar{m}_{0}, \infty\right)$. For the input nonlinearity, it is clear from (43) that $l=3$ and $\left|h_{3}\right| \geq 0.3$. Therefore, $\beta$ was chosen to be $10^{-3}$. For the controller, $h(v)$ was chosen to be the identity yielding $\lambda=1$. Since (27) is cubic, it can have more than one real root, and hence, the controller can track any one of the real roots.

We next present simulation and experimental results for the adaptive feedback linearization controller that adapts to both the unknown inertia and the unknown input nonlinearity. The control parameters in the adaptive control law are chosen to be $k=70, q=5$, and $G=I_{4 \times 4}$. Fig. 18 shows simulation results for tracking the command signal $\nu(t)$. The simulated angular velocity tracking error given by $\tilde{\omega}(t)$ is shown in Fig. 19 .

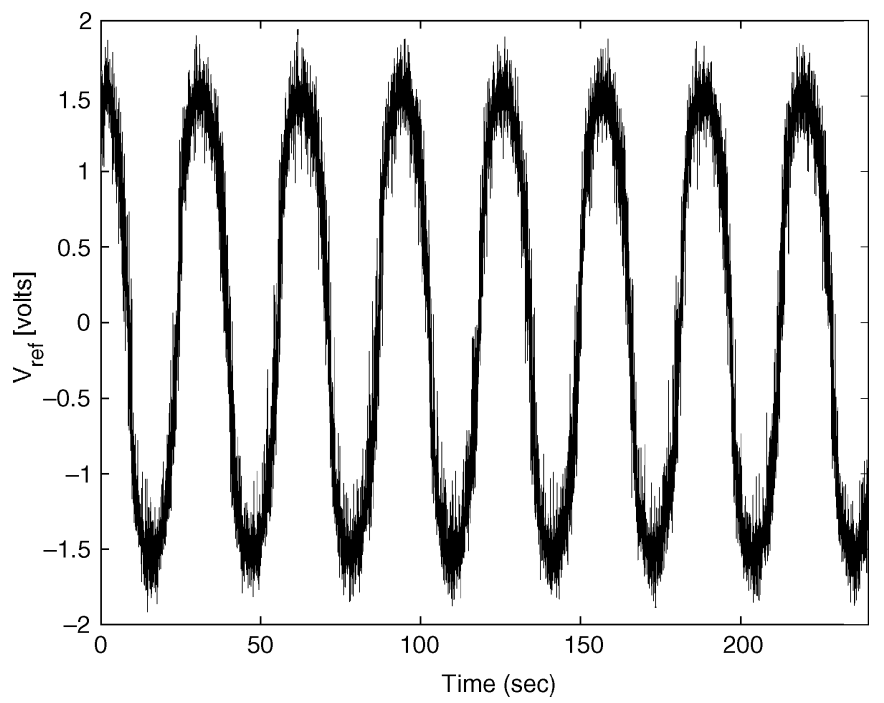

Fig. 17. Experimental control signal with inverted actuator nonlinearity.

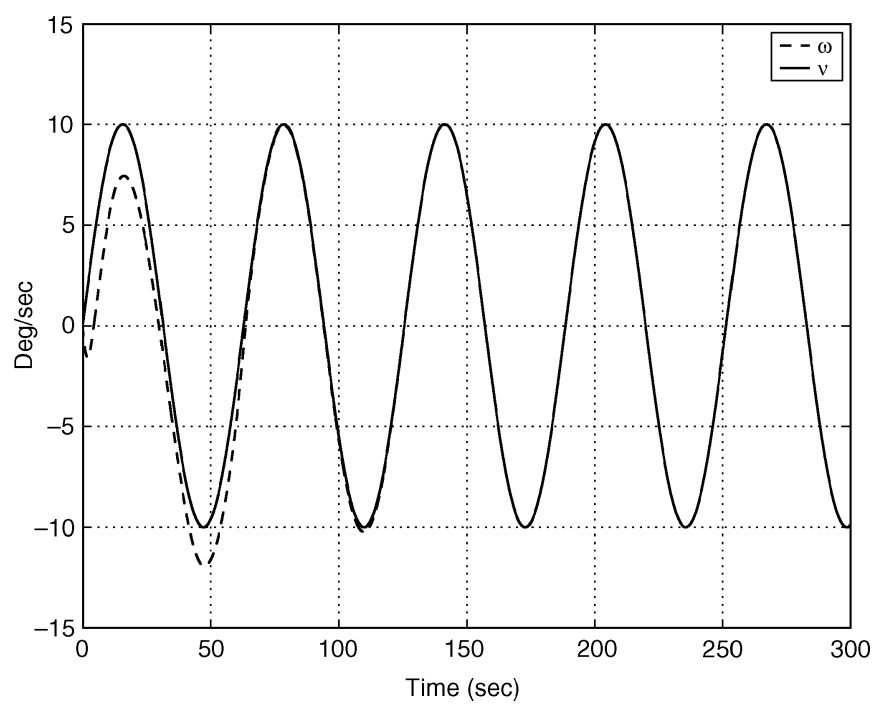

Fig. 18. Simulated angular velocities $\omega(t)$ and $\nu(t)$ using adaptive feedback linearization for $\nu(t)=10 \sin (0.1 t)^{\circ} / \mathrm{s}$.

The simulation results in Fig. 19 for the adaptive feedback linearization controller for the nonaffine system with (43) show that the angular velocity tracking error converges to zero. By comparison, Figs. 10 and 13 show that residual oscillations remain in cases 2 and 3 . In case 3 , better results can be obtained if the exact inverse of (43) is chosen rather than the piecewise linear approximation. However, in either case, this method requires knowledge of the input nonlinearity. In contrast, the adaptive feedback linearization controller does not require knowledge of the input nonlinearity.

Next, the adaptive feedback linearization controller is implemented on the Triaxial Attitude Control Testbed. The angular velocity is plotted in Fig. 20, while the angular velocity tracking error $\tilde{\omega}(t)$ is plotted in Fig. 21. As can be seen, the angular velocity $\omega(t)$ tracks the command signal $\nu(t)$, and the angular velocity error $\tilde{\omega}(t)$ converges to zero.

In Figs. 22 and 23, experimental anomalies can be seen. In particular, a transient excursion away from zero occurs at around $300 \mathrm{~s}$ in both plots. The system soon recovers, and 


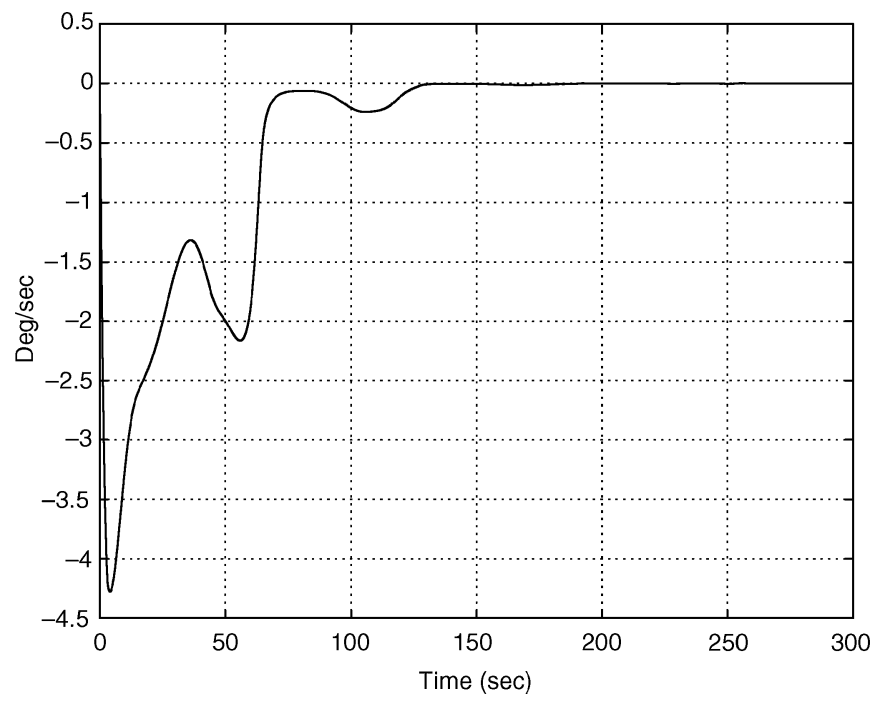

Fig. 19. Simulated angular velocity error $\tilde{\omega}$ with adaptive feedback linearization for $\nu(t)=10 \sin (0.1 t)^{\circ} / \mathrm{s}$.

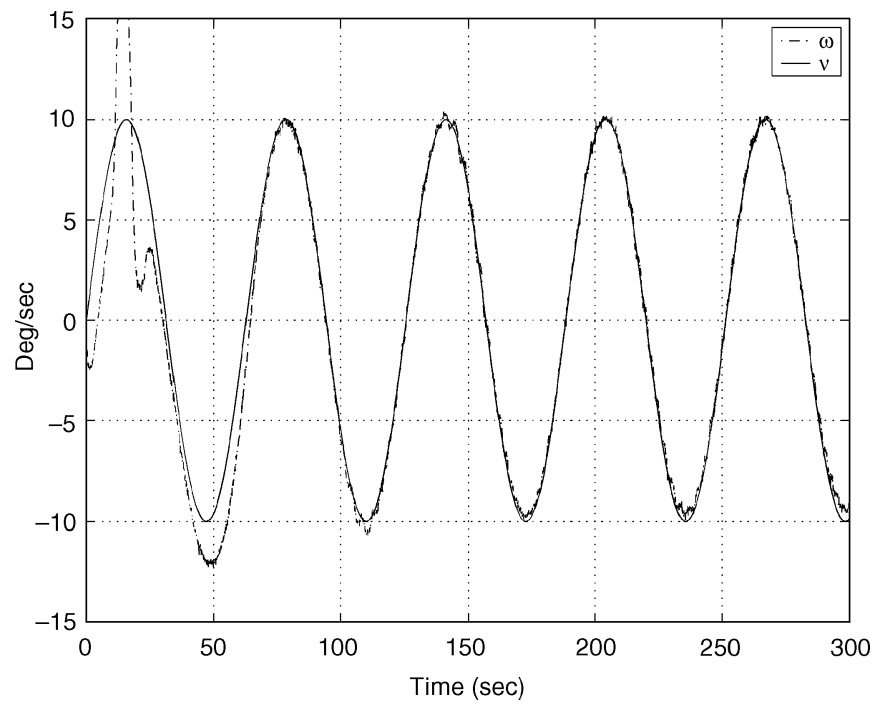

Fig. 20. Experimental values for angular velocities $\omega(t)$ and $\nu(t)$ using adaptive feedback linearization for $\nu(t)=10 \sin (0.1 t)^{\circ} / \mathrm{s}$.

the angular velocity error $\tilde{\omega}(t)$ converges to zero again. This behavior is similar to the phenomenon of bursting observed in other adaptive algorithms [25], [26]. One possible explanation for the bursting is the presence of noise in the output signal $\omega(t)$. Specifically, if $\omega(t)=\omega_{\mathrm{t}}(t)+d(t)$, where $\omega_{\mathrm{t}}(t)$ is the true value of $\omega(t)$ and $d(t)$ is the noise present in the signal, then $\dot{V}(\cdot)$ contains the term $\tilde{M}^{\mathrm{T}} \sigma(v) d(t)$, where $v$ is a real solution of

$$
v+(\tilde{M}+M)^{\mathrm{T}} \sigma(v)=-k(\tilde{\omega}+d)+\dot{\nu}(\tilde{J}+J) .
$$

In (48), the noise becomes significant for small values of $\tilde{\omega}$. Since $v$ might not depend continuously on $d(t)$, the magnitude of $\tilde{M}^{\mathrm{T}} \sigma(v) d(t)$ can change discontinuously by a large value even for small variations in $d(t)$, causing $\dot{V}(\cdot)$ to jump discontinuously to a large positive value.

To test the hypothesis that the bursting in the angular velocity error is caused by noisy measurements, simulations were performed with artificial noise added to the output $\omega(t)$. Figs. 24

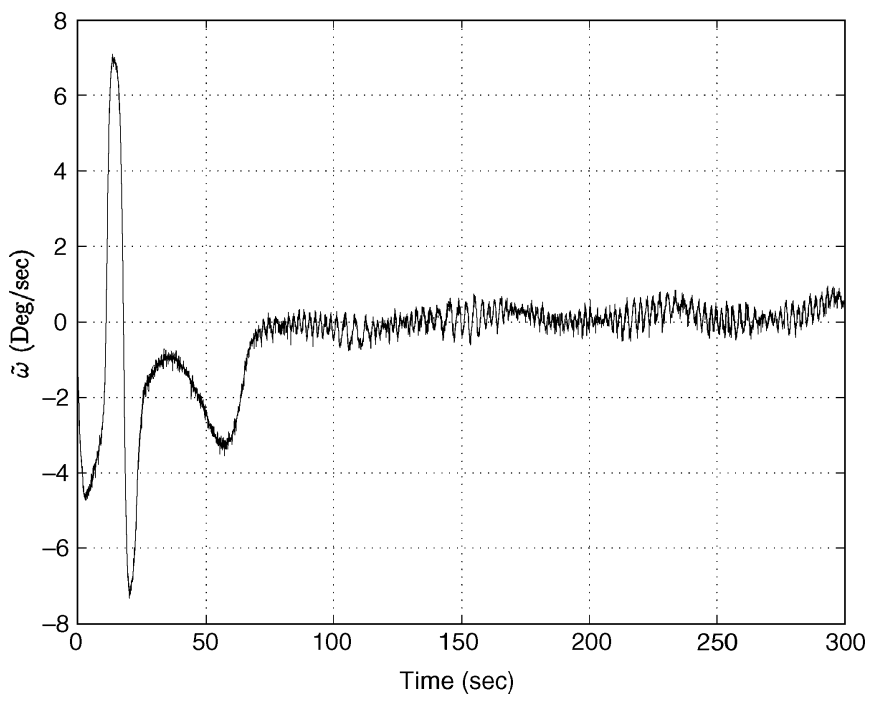

Fig. 21. Experimental values for angular velocity error $\tilde{\omega}$ with adaptive feedback linearization for $\nu(t)=10 \sin (0.1 t)^{\circ} / \mathrm{s}$.

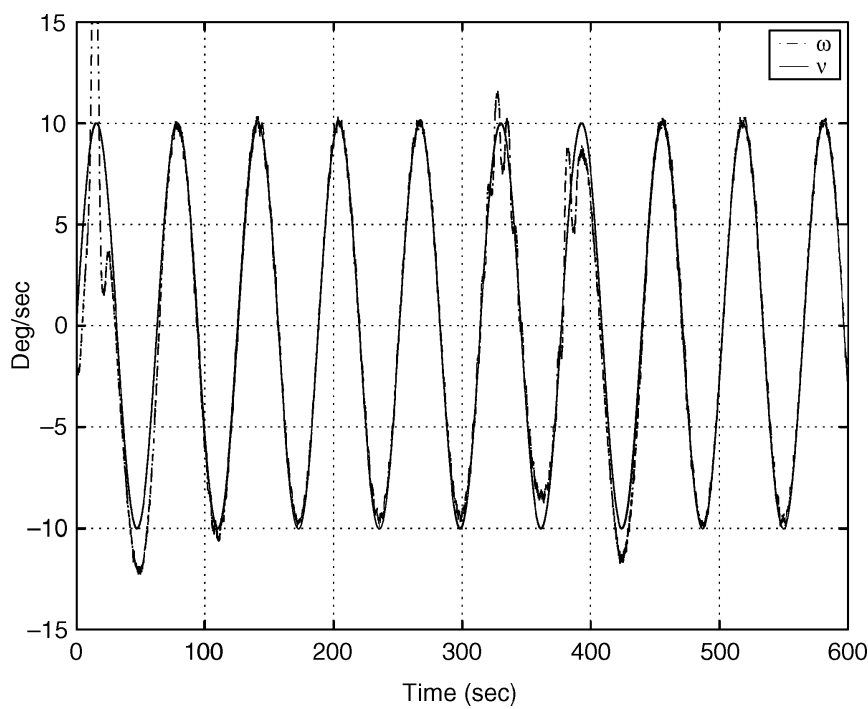

Fig. 22. Experimental values for angular velocities $\omega(t)$ and $\nu(t)$ using adaptive feedback linearization for $\nu(t)=10 \sin (0.1 t)^{\circ} / \mathrm{s}$.

and 25 show simulation plots for tracking of $\nu(t)$ and angular velocity error $\tilde{\omega}(t)$, respectively, for the plant with noise at the output. The similarity between Figs. 22 and 24 and between Figs. 23 and 25 suggest that the bursting in the output is due to noisy angular velocity measurements. A possible solution to the problem of bursting is to use a deadzone to disable adaptation when the angular velocity error $\tilde{\omega}(t)$ drops below a chosen threshold [27], [28].

\section{CONCLUSION}

An adaptive feedback control algorithm is developed to provide global tracking of commanded angular velocity signals for a planar, rigid body which has an input nonlinearity. The first result is for the design of an adaptive controller assuming an affine control input. This controller assumes no prior knowledge of the inertia and is, thus, unconditionally robust with respect to this parametric uncertainty with global convergence. It is shown 


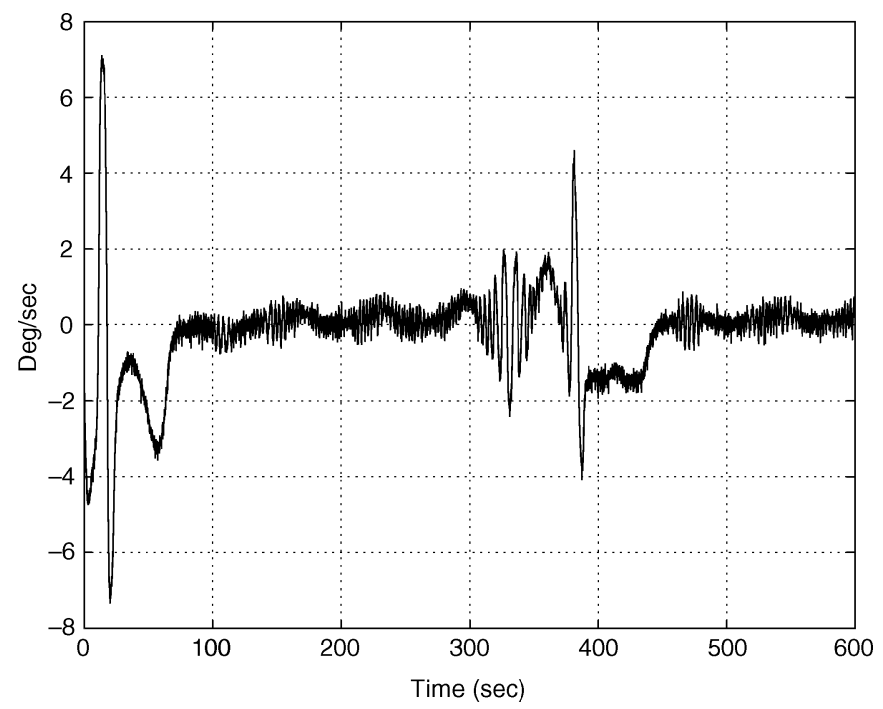

Fig. 23. Experimental values for angular velocity error $\tilde{\omega}(t)$ with adaptive feedback linearization for $\nu(t)=10 \sin (0.1 t)^{\circ} / \mathrm{s}$.

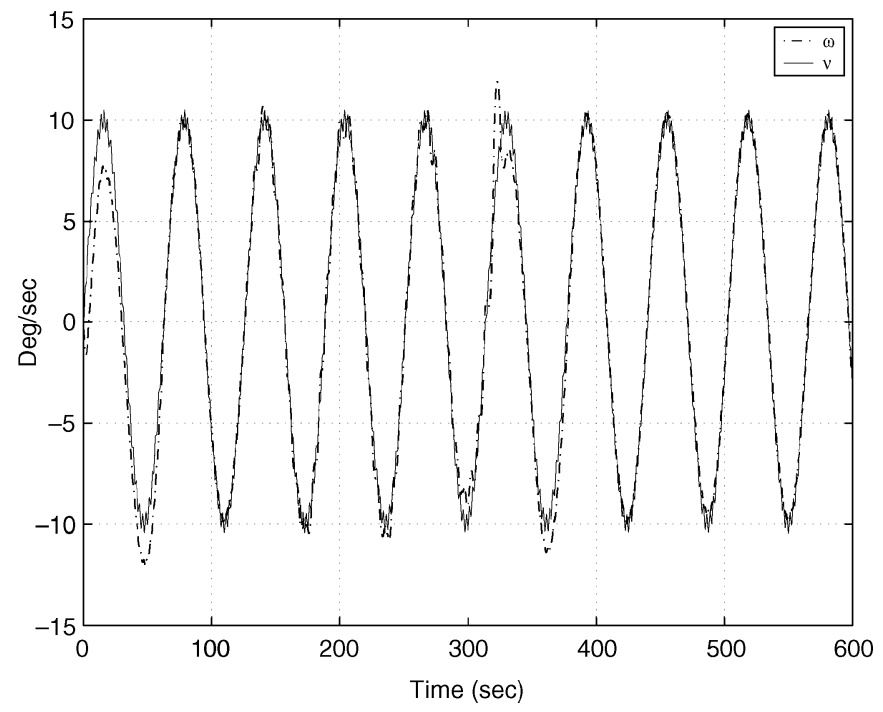

Fig. 24. Simulated angular velocities $\omega(t)$ and $\nu(t)$ using adaptive feedback linearization for $\nu(t)=10 \sin (0.1 t)^{\circ} / \mathrm{s}$ with sensor noise effect.

using Lyapunov methods that the angular velocity tracking error converges to zero. Furthermore, the control algorithm is used to identify the inertia when the commanded angular velocity signal has a piecewise continuous derivative and does not converge to zero. The command signal need not be bounded. Numerical simulations demonstrate tracking and identification of the inertia.

Next, the design of an adaptive controller including an unknown input nonlinearity is developed. Precise conditions for the convergence of the angular velocity error using Lyapunov analysis have been presented. Four different controllers have been implemented on the Triaxial Attitude Control Testbed for planar rotation, and their performance is compared. Improved results for angular velocity tracking are achieved for constant and sinusoidal command signals using the adaptive control algorithm that ignores the input nonlinearity, compared to the proportional controller. Inertia estimates are obtained using the same adaptive controller for command signals with characteristics as mentioned above. An actuator input nonlinearity is

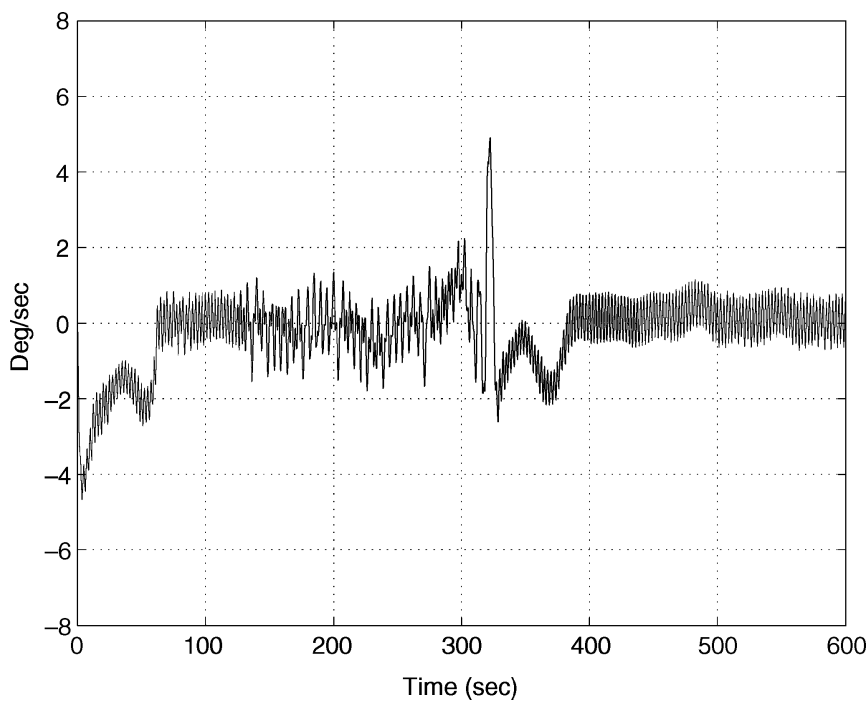

Fig. 25. Simulated angular velocity error $\tilde{\omega}(t)$ with adaptive feedback linearization for $\nu(t)=10 \sin (0.1 t) \%$ s with sensor noise effect.

identified and its effects are studied by simulation. A piecewise linear approximation of the nonlinearity is inverted and this inverse is experimentally found to improve angular velocity tracking by $37.5 \%$ and inertia identification by $33.3 \%$ for sinusoidal commands.

Finally, the adaptive feedback linearization controller is used to compensate for the unknown input nonlinearity and the resulting controller is tested on the Triaxial Attitude Control Testbed. Simulation and experimental results provide validation of the effectiveness of the adaptive technique and highlight the effect of sensor noise.

\section{APPENDIX A}

Lemma 1: Let $g:[0, \infty) \mapsto \mathbb{R}$ and $f:[0, \infty) \mapsto \mathbb{R}$ and assume that $\lim _{t \rightarrow \infty} f(t)$ exists and

$$
\lim _{t \rightarrow \infty} g(t) f(t)=0 \text {. }
$$

Furthermore, suppose that either $\lim _{t \rightarrow \infty} g(t) \neq 0$ or $\lim _{t \rightarrow \infty} g(t)$ does not exist. Then, $\lim _{t \rightarrow \infty} f(t)=\stackrel{t \rightarrow}{0 \text {. }}$

Proof: First, consider the case in which $\lim _{t \rightarrow \infty} g(t) \neq 0$. Then, $0=\lim _{t \rightarrow \infty} g(t) f(t)=\lim _{t \rightarrow \infty} g(t) \lim _{t \rightarrow \infty} f(t)$. Hence, $\lim _{t \rightarrow \infty} f(t)=0$.

Next, let $\bar{f} \triangleq \lim _{t \rightarrow \infty} f(t)$ and assume that $\lim _{t \rightarrow \infty} g(t)$ does not exist. Since $\lim _{t \rightarrow \infty} f(t)$ exists, it follows that, for every $\epsilon_{1}>0$, there exists $t_{1}>0$ such that, for all $t \geq t_{1},|f(t)-\bar{f}|<\epsilon_{1}$. Similarly, it follows from (49) that, for every $\epsilon_{2}>0$, there exists $t_{2}>0$ such that, for all $t \geq t_{2},|g(t) f(t)|<\epsilon_{2}$. Now, since $\lim _{t \rightarrow \infty} g(t)$ does not exist, there exists a positive constant $\kappa$ such that, for every $T>0$, there exists $T_{1} \geq T$ such that $\left|g\left(T_{1}\right)\right| \geq \kappa$.

Now, assume that $\bar{f} \neq 0$ and let $0<\epsilon_{1} \leq|\bar{f}| / 2$. Then, there exists $t_{1}>0$ such that, for all $t \geq t_{1},|f(t)-\bar{f}|<$ $\epsilon_{1} \leq|\bar{f}| / 2$. Thus, $|f(t)|>|\bar{f}| / 2$ for all $t \geq t_{1}$. Therefore, for all $t>t_{1},|g(t) f(t)| \geq|g(t)||\bar{f}| / 2$. Now, choose $0<\epsilon_{2} \leq$ $|\bar{f}| \kappa / 4$. Then, (49) implies there exists $t_{2}>0$ such that, for all $t \geq t_{2},|g(t) f(t)|<\epsilon_{2} \leq|\bar{f}| \kappa / 4$. Defining $t_{3} \triangleq \max \left\{t_{1}, t_{2}\right\}$, 
it follows that, for all $t>t_{3},|g(t)||\bar{f}| / 2 \leq|g(t) f(t)|<|\bar{f}| \kappa / 4$. Thus, for all $t>t_{3},|g(t)|<\kappa / 2$. However, there exists $T_{1} \geq t_{3}$ such that $\left|g\left(T_{1}\right)\right| \geq \kappa$, which is a contradiction. Hence $\bar{f}=0$.

\section{APPENDIX B}

Definition 1: Let $\psi:[0, \infty) \mapsto \mathbb{R}^{n}$ and let $\left\{t_{i}\right\}_{i=1}^{\infty}$ be an increasing sequence in $[0, \infty)$ such that inf $i_{i \in \mathcal{Z}_{+}}\left(t_{i+1}-t_{i}\right)>0$. Let $\|\cdot\|$ denote a norm on $\mathbb{R}^{n}$. Then $\psi$ is globally piecewise uniformly continuous if there exists $\delta:[0, \infty) \mapsto[0, \infty)$ such that, for every $i \in \mathcal{Z}_{+}$and $\epsilon>0,\left\|\psi(t)-\psi(t)^{\prime}\right\|<\epsilon$ for all $t$, $t^{\prime} \in\left[t_{i}, t_{i+1}\right)$, such that $\left|t-t^{\prime}\right|<\delta(\epsilon)$.

Lemma 2: Let $\psi:[0, \infty) \mapsto \mathbb{R}^{n}$ be globally piecewise uniformly continuous and assume that $\int_{0}^{\infty} \psi(\tau) d \tau$ exists. Then $\psi(t) \rightarrow 0$ as $t \rightarrow \infty$.

Proof: It suffices to consider $n=1$. Suppose $\psi(t)$ does not converge to zero as $t \rightarrow \infty$. Then, there exists a positive constant $k$ such that, for every $T>0$, there exists $T_{1}>T$ such that $\left|\psi\left(T_{1}\right)\right| \geq k$.

Next, let $\left\{t_{i}\right\}_{i=1}^{\infty}$ be the increasing sequence of points of discontinuity of $\psi$ as in Definition 1. Let $\mathcal{T} \triangleq \inf _{i \in \mathcal{Z}_{+}}\left(t_{i+1}-t_{i}\right)$, and let $j:[0, \infty) \mapsto \mathcal{Z}_{+}$be given by

$$
j(t) \triangleq i, \quad t_{i} \leq t<t_{i+1}, i \in \mathcal{Z}_{+} .
$$

The function $j(t)$ gives the index of the interval in which $t$ resides. Thus, $T_{1} \in\left[t_{j\left(T_{1}\right)}, t_{j\left(T_{1}\right)+1}\right)$. Furthermore, note that either $T_{1}-t_{j\left(T_{1}\right)}<t_{j\left(T_{1}\right)+1}-T_{1}$ or $T_{1}-t_{j\left(T_{1}\right)} \geq t_{j\left(T_{1}\right)+1}-T_{1}$.

Assume that $T_{1}-t_{j\left(T_{1}\right)}<t_{j\left(T_{1}\right)+1}-T_{1}$. Now, since $\psi(t)$ is globally piecewise uniformly continuous, there exists $\delta \in$ $(0, \mathcal{T} / 2]$ such that, for every $\tau \in(0, \delta],\left|\psi\left(T_{1}+\tau\right)-\psi\left(T_{1}\right)\right|<$ $(k / 2)$. Hence, for every $t \in\left[T_{1}, T_{1}+\delta\right]$

$$
\begin{aligned}
|\psi(t)| & =\left|\psi(t)-\psi\left(T_{1}\right)+\psi\left(T_{1}\right)\right|, \\
& \geq\left|\psi\left(T_{1}\right)\right|-\left|\psi(t)-\psi\left(T_{1}\right)\right|, \\
& >k-\frac{k}{2}=\frac{1}{2} k .
\end{aligned}
$$

Note that $\psi(t)$ has the same sign for all $t \in\left[T_{1}, T_{1}+\delta\right]$. Thus

$$
\left|\int_{T_{1}}^{T_{1}+\delta} \psi(t) d t\right|=\int_{T_{1}}^{T_{1}+\delta}|\psi(t)| d t>\frac{1}{2} k \delta .
$$

It can similarly be shown that, if $T_{1}-t_{j\left(T_{1}\right)} \geq t_{j\left(T_{1}\right)+1}-T_{1}$, then there exists $\delta \in(0, \mathcal{T} / 2]$ such that

$$
\left|\int_{T_{1}-\delta}^{T_{1}} \psi(t) d t\right|>\frac{1}{2} k \delta
$$

Hence, denoting $g(t) \triangleq \int_{0}^{t} \psi(t) d t$, (52) and (53) imply that, for every $T>0$, there exist $T_{1} \geq T$ and positive constants $k$ and $\delta \in(0, \mathcal{T} / 2]$ such that either

$$
\left|g\left(T_{1}+\delta\right)-g\left(T_{1}\right)\right|>\frac{1}{2} k \delta
$$

or

$$
\left|g\left(T_{1}-\delta\right)-g\left(T_{1}\right)\right|>\frac{1}{2} k \delta .
$$

Therefore, $\lim _{t \rightarrow \infty} g(t)$ does not exist, which is a contradiction.

\section{APPENDIX C}

Lemma 3: Consider (18) and (19) where $h: \mathbb{R} \mapsto \mathbb{R}$ is an onto polynomial of order $l$ and $\hat{h}: \mathbb{R} \mapsto \mathbb{R}$ is given by $\hat{h}(u)=$ $\lambda u$, where $\lambda \in \mathbb{R} \backslash\{0\}$. Then $l$ is odd and

$$
\Delta\left(\hat{h}^{-1}(v)\right)=M^{\mathrm{T}} \sigma(v)
$$

for all $v \in \mathbb{R}$, where $\sigma(v)=\left[v^{l} v^{l-1} \ldots v 1\right]^{\mathrm{T}}$ and $M \in \mathbb{R}^{l+1}$.

Proof: Suppose $l$ is even. Then

$$
\lim _{v \rightarrow \infty} h(v)=\lim _{v \rightarrow-\infty} h(v)=\infty .
$$

Since $h$ is onto, there exists $v_{\infty} \in \mathbb{R}$ such that $\lim _{v \rightarrow v_{\infty}} h(v)=$ $-\infty$. Furthermore, (54) yields that $v_{\infty} \neq \pm \infty$. Therefore, it follows from intermediate value theorem that there exist $x_{1} \in$ $\left(-\infty, v_{\infty}\right)$ and $x_{2} \in\left(v_{\infty}, \infty\right)$, such that $h\left(x_{1}\right)=h\left(x_{2}\right)=0$.

Next, consider the restriction of $h:\left[x_{1}, x_{2}\right] \mapsto \mathbb{R}$. Since $h$ is continuous and $\left[x_{1}, x_{2}\right]$ is compact, $h(x)$ attains a minimum on $x \in\left[x_{1}, x_{2}\right]$. However, $v_{\infty} \in\left[x_{1}, x_{2}\right]$, and $\lim _{v \rightarrow v_{\infty}} h(v)=-\infty$, which is a contradiction. Hence, $l$ is odd.

Now, from (18) and (19), it follows that $\Delta\left(\hat{h}^{-1}(v)\right)=$ $h\left(\hat{h}^{-1}(v)\right)-v=h(v / \lambda)-v$. Since $h$ is an odd order polynomial function, $\Delta(\hat{h}(v))$ is a polynomial of order less than or equal to $l$. The result then follows.

Lemma 4: Consider the algebraic (27) where $\hat{M} \in \mathbb{R}^{l+1}$. Denote $\hat{M} \triangleq\left[\hat{m}_{0} \hat{M}_{l}^{\mathrm{T}}\right]^{\mathrm{T}}$, where $\hat{m}_{0} \in \mathbb{R}$ and $\hat{M}_{l} \in \mathbb{R}^{l}$. Then for all $\hat{m}_{0} \in\left[\bar{m}_{0}, \infty\right)$ where $\bar{m}_{0}$ is defined in (28), $\hat{M}_{l} \in \mathbb{R}^{l}$ and $v_{a} \in \mathbb{R},(27)$ has at least one real solution.

Proof: Consider (27) for the case $l=1$. Then, (27) is equivalent to

$$
\left(1+\hat{m}_{0}\right) v+\hat{M}_{1}=v_{a} .
$$

Since, $\hat{m}_{0} \in\left[\bar{m}_{0}, \infty\right)$ and $\bar{m}_{0}=(\beta /|\lambda|)-1$, therefore, $(1+$ $\left.\hat{m}_{0}\right) \geq(\beta /|\lambda|)>0$. Thus, $v=v_{a}-\hat{M}_{1} / 1+\hat{m}_{0}$ is a solution of (27).

Next, consider the case $l>1$. Then (27) is equivalent to

$$
\hat{m}_{0} v^{l}+\hat{M}_{l}^{\mathrm{T}} \sigma_{l}(v)+v=v_{a} .
$$

Since $\hat{m}_{0} \in\left[\bar{m}_{0}, \infty\right)$ and $\bar{m}_{0}=\beta /\left|\lambda^{l}\right|$, therefore $\hat{m}_{0} \geq$ $\left(\beta /\left|\lambda^{l}\right|\right)>0$. Thus, since (55) is an odd order polynomial, there exists a real root of (55). Thus, (27) has a solution. Therefore, for all $\hat{m}_{0} \in\left[\bar{m}_{0}, \infty\right), \hat{M}_{l} \in \mathbb{R}^{l}$ and $v_{a} \in \mathbb{R}$, the algebraic (27) has a solution $v \in \mathbb{R}$.

Lemma 5: Consider the closed-loop (31)-(35). Assume that (31)-(34) has a local generalized solution for all $\tilde{\omega}(0), \tilde{J}(0)$, $\tilde{M}_{l}(0), \hat{m}_{0}(0) \in\left[\bar{m}_{0}, \infty\right)$, and $v \in \mathbb{R}$, where $\bar{m}_{0}$ is given by (28) and $\nu$ is $C^{1}$ and $\dot{\nu}$ is bounded. Choose $\hat{m}_{0}(0) \in\left[\bar{m}_{0}, \infty\right)$. If $\nu$ is $C^{1}$ and $\dot{\nu}$ is bounded, then the closed-loop dynamics of (31)-(35) has a generalized solution that satisfies $\hat{m}_{0}(t) \in$ $\left[\bar{m}_{0}, \infty\right)$ over some maximal interval of existence $[0, T)$.

Proof: We first show that whenever the solution of the closed-loop (31)-(35) exists for $t \geq 0, \hat{m}_{0}(t) \in\left[\bar{m}_{0}, \infty\right)$, and a solution $v \in \mathbb{R}$ of (35) exists. Hence, the solution remains contained in the domain where local existence of a generalized solution of (31)-(34) is guaranteed. Then, by standard continuation arguments and local existence of a generalized solution 
of (31)-(34), it follows that there exists some maximal interval $[0, T)$, such that the closed-loop (31)-(35) has a generalized solution for all $t \in[0, T)$. Clearly, it satisfies $\hat{m}_{0}(t) \in\left[\bar{m}_{0}, \infty\right)$ for all $t \in[0, T)$.

Whenever the solution of the closed-loop (31)-(35) exists, the first condition $\hat{m}_{0}(t) \in\left[\bar{m}_{0}, \infty\right)$ for all $t \geq 0$ follows from the fact that $\hat{m}_{0}(0) \in\left[\bar{m}_{0}, \infty\right)$ and $\dot{\hat{m}}_{s} \geq 0$ at $\hat{m}_{0}=\bar{m}_{0}$. To see this, consider (29). If $\hat{m}_{0}=\bar{m}_{0}$, then either $\dot{\hat{m}}_{s}=0$ for $\tilde{\omega} v^{l}<0$ or $\dot{\hat{m}}_{s}=g \tilde{\omega} v^{l}$ for $\tilde{\omega} v^{l} \geq 0$ and hence, $\dot{\hat{m}}_{s} \geq 0$.

Whenever the solution of the closed-loop (31)-(35) exists, the second condition that the algebraic (35) has a solution for all $t \geq 0$ follows from identifying (35) with (27) and combining the result from Lemma 4 and the condition that $\hat{m}_{0}(t) \in\left[\bar{m}_{0}, \infty\right)$ for all $t \geq 0$. The result then follows.

Lemma 6: Assume that Assumptions 1 and 2 hold true and define $\hat{h}: \mathbb{R} \mapsto \mathbb{R}$ as $\hat{h}(u)=\lambda u$, where $\lambda \in \backslash \mathbb{R}\{0\}$ satisfies $\operatorname{sign}(\lambda)=\operatorname{sign}\left(h_{l}\right)$. Consider (17), (18), (19), (23), and (28). Then, $\bar{m}_{0}-m_{0} \leq 0$, where $M=\left[m_{0} M_{l}^{\mathrm{T}}\right]^{\mathrm{T}}$.

Proof: From (23) and (19), note that $\Delta(\hat{h}(v))=m_{0} v^{l}+$ $M_{l}^{\mathrm{T}} \sigma_{l}(v)=h\left(\hat{h}^{-1}(v)\right)-v$, where $\sigma_{l}(v)=\left[v^{l-1} \ldots v 1\right]^{\mathrm{T}}$ and $v \in \mathbb{R}$.

First consider the case $l=1$. Therefore, from Assumption 1, $h(u)=h_{1} u+h_{0}$ and $\left|h_{1}\right| \geq \beta$. Thus

$\Delta\left(\hat{h}^{-1}(v)\right)=m_{0} v+M_{1}=h\left(\frac{v}{\lambda}\right)-v=\left(\frac{h_{1}}{\lambda}-1\right) v+h_{0}$.

Therefore, $m_{0}=\left(h_{1} / \lambda\right)-1$. Since $\operatorname{sign}(\lambda)=\operatorname{sign}\left(h_{1}\right)$ and $\left|h_{1}\right| \geq \beta$

$$
\frac{h_{1}}{\lambda}=\frac{\left|h_{1}\right| \operatorname{sign}\left(h_{1}\right)}{|\lambda| \operatorname{sign}(\lambda)} \geq \frac{\beta}{|\lambda|} .
$$

Thus $m_{0}=\left(h_{1} / \lambda\right)-1 \geq(\beta /|\lambda|)-1=\bar{m}_{0}$ from (28) and hence, $m_{0} \geq \bar{m}_{0}$.

For $l>1, h(u)=h_{l} u^{l}+h_{l-1} u^{l-1}+\ldots+h_{1} u+h_{0}$, where $\left|h_{l}\right| \geq \beta$. Thus,

$$
\begin{aligned}
\Delta\left(\hat{h}^{-1}(v)\right) & =m_{0} v^{l}+M_{l}^{\mathrm{T}} \sigma_{l}(v)=h\left(\frac{v}{\lambda}\right)-v \\
& =\frac{h_{l}}{\lambda^{l}} v^{l}+\frac{h_{l-1}}{\lambda^{l-1}} v^{l-1}+\ldots+\left(\frac{h_{1}}{\lambda}-1\right) v+h_{0} .
\end{aligned}
$$

Therefore, $m_{0}=h_{l} / \lambda^{l}$. Since $l$ is odd and $\operatorname{sign}(\lambda)=\operatorname{sign}\left(h_{l}\right)$

$$
\frac{h_{l}}{\lambda^{l}}=\frac{\left|h_{l}\right| \operatorname{sign}\left(h_{l}\right)}{\left|\lambda^{l}\right| \operatorname{sign}(\lambda)} \geq \frac{\beta}{\left|\lambda^{l}\right|} .
$$

Therefore, $m_{0}=\left(h_{l} / \lambda^{l}\right) \geq\left(\beta /\left|\lambda^{l}\right|\right)=\bar{m}_{0}$ from (28) and hence, $m_{0} \geq \bar{m}_{0}$. Thus, $\bar{m}_{0}-m_{0} \leq 0$.

\section{ACKNOWLEDGMENT}

The authors would like to thank Prof. N. Hovakimyan for helpful discussions concerning adaptive feedback linearization.

\section{REFERENCES}

[1] R. W. Brockett, "Asymptotic stability and feedback stabilization," in Differential Geometric Control Theory, R. W. Brockett, R. S. Millman, and H. J. Sussmann, Eds., 1983, vol. 27, Progress in Mathematics, pp. 181-191.

[2] D. Aeyels and M. Szafranski, "Comments on the stabilizability of the angular velocity of a rigid body," Sys. Contr. Lett., vol. 10, no. 1, pp. 35-39, Jan. 1988

[3] E. D. Sontag and H. J. Sussmann, "Further comments on the stabilizability of the angular velocity of a rigid body," Sys. Contr. Lett., vol. 12, no. 3, pp. 213-217, Apr. 1989.

[4] C. I. Byrnes and A. Isidori, "New results and examples in nonlinear feedback stabilization," Sys. Contr. Lett., vol. 12, no. 5, pp. 437-442, Jun. 1989.

[5] C.-J. Wan and D. Bernstein, "Nonlinear feedback control with global stabilization," Dyn. Control, vol. 5, no. 4, pp. 321-346, Oct. 1995.

[6] R. Outbib and G. Sallet, "Stabilizability of the angular velocity of a rigid body revisited," Sys. Contr. Lett., vol. 18, no. 2, pp. 93-98, Feb. 1992.

[7] J. L. Junkins and J. D. Turner, Optimal Spacecraft Rotational Maneuvers. New York: Elsevier, 1985.

[8] K. D. Bilimoria and B. Wie, "Time-optimal three-axis reorientation of a rigid spacecraft," J. Guid. Contr. Dyn., vol. 16, no. 3, May/Jun. 1993.

[9] T. A. W. Dwyer and A. L. Batten, "Exact spacecraft detumbling and reorientation maneuvers with gimbaled thrusters and reaction wheels," Astronautical Sci., vol. 33, no. 2, pp. 217-232, Apr./Jun. 1983.

[10] J. Dzielski, E. Bergmann, J. Paradiso, D. Rowell, and D. Wormley, "Approach to control moment gyroscope steering using feedback linearization," J. Guid. Contr., vol. 14, no. 1, pp. 96-106, Jan./Feb. 1991.

[11] N. A. Chaturvedi et al., "Globally convergent adaptive tracking of angular velocity and inertia identification of a 3 DOF rigid body," IEEE Trans. Contr. Syst. Technol., to be published.

[12] S. S. Sastry and A. Isidori, "Adaptive control of linearizable systems," IEEE Trans. Autom. Control, vol. 34, no. 11, pp. 1123-1131, Nov. 1989.

[13] M. Krstić, I. Kanellakopoulos, and P. V. Kokotović, Nonlinear and Adaptive Control. New York: Wiley, 1995.

[14] H. G. Kwatny and G. L. Blankenship, Nonlinear Control and Analytical Mechanics: A Computational Approach. Boston, MA: Birkhäauser, 2000.

[15] T. Zhang, S. S. Ge, and C. C. Hang, "Direct adaptive control of nonaffine nonlinear systems using multilayer neural networks," in Proc. Amer. Contr. Conf., 1998, pp. 515-519.

[16] S. S. Ge, T. H. Lee, and J. Wang, "Adaptive control of nonaffine nonlinear systems using neural networks," in Proc. IEEE Int. Symp. Intelligent Contr., 2000, pp. 13-18.

[17] F. Nardi and A. J. Calise, "Robust adaptive nonlinear control using single hidden layer neural networks," in Proc. 39th IEEE Conf. Dec. Contr., 2000, pp. 3825-3830.

[18] N. Hovakimyan, F. Nardi, and A. J. Calise, "A novel error observerbased adaptive output feedback approach for control of uncertain systems," IEEE Trans. Autom. Contr., vol. 47, no. 8, pp. 1310-1314, Aug. 2002.

[19] A. F. Filippov, Differential Equations with Discontinuous Righthand Sides. Norwell, MA: Kluwer, 1988.

[20] O. Hájek, "Discontinuous differential equations-I, II," J. Differ. Equ., vol. 32, no. 2, pp. 149-185, May 1979.

[21] H. K. Khalil, Nonlinear Systems. Englewood Cliffs, NJ: Prentice-Hall, 2002.

[22] N. Hovakimyan, F. Nardi, A. Calise, and N. Kim, "Adaptive output feedback control of uncertain nonlinear systems using single hidden layer neural networks," IEEE Trans. Neural Netw., vol. 13, no. 6, pp. 1420-1431, Nov. 2002.

[23] J. P. Aubin and A. Cellina, Differential Inclusions—Set Valued Maps and Viability Theory. New York: Springer-Verlag, 1984.

[24] M. Kunze, Non-Smooth Dynamical Systems. Berlin, Germany: Springer, 2000.

[25] B. A. Huberman and E. Lumer, "Dynamics of adaptive systems," IEEE Trans. Circuits Syst., vol. 37, no. 4, pp. 547-550, 1990.

[26] G. J. Rey, R. R. Bitmead, and C. R. Johnson, "The dynamics of bursting in simple adaptive feedback systems with leakage," IEEE Trans. Circuits Syst., vol. 38, no. 5, pp. 476-488, May 1991.

[27] J. M. Krause and M. J. Englehart, "An adaptive deadzone technique with a burst alleviation example," IEEE Trans. Autom. Contr., vol. 38, no. 9, pp. 1405-1409, Sep. 1993.

[28] K. S. Tsakalis, "Performance limitations of adaptive parameter estimation and system identification algorithms in the absence of excitation," Automatica, vol. 32, no. 4, pp. 549-560, Apr. 1996. 


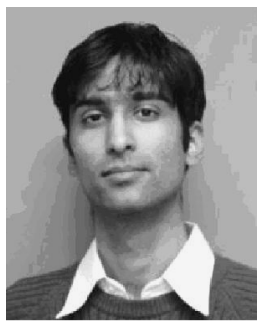

Nalin A. Chaturvedi received the B.Tech. and M.Tech. degrees in aerospace engineering from the Indian Institute of Technology, Bombay, India, in 2003, where he received the Institute Silver Medal. He is currently pursuing the Ph.D. degree in aerospace engineering at the University of Michigan, Ann Arbor.

$\mathrm{He}$ is a Research Fellow with the University of Michigan. His main research interests include nonlinear stability theory with applications to aerospace and mechanical systems/robotics, geometric mechanics, nonlinear and geometric control, nonlinear dynamical systems, state estimation, and adaptive control with applications to nonaffine systems.

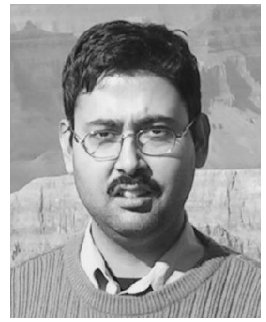

Amit K. Sanyal received the B.Tech. degree in aerospace engineering from the Indian Institute of Technology, Kanpur, India, in 1999, and the M.S. degree in aerospace engineering from Texas A\&M University, College Station, in 2001. He also received the M.S. degree in mathematics and the $\mathrm{Ph} . \mathrm{D}$. degree in aerospace engineering from the University of Michigan, Ann Arbor, in 2004

In 2004, he became a Post-Doctoral Research Fellow with teaching duties, in the Mechanical and Aerospace Engineering Department at Arizona State University, Tempe. His main research interests include geometric mechanics, nonlinear and geometric control, nonlinear dynamical systems, mechatronics, variational integration of Lagrangian/Hamiltonian systems, optimal control and estimation, and applications of geometric/algebraic methods to dynamical systems.

Dr. Sanyal was a recipient of the College of Engineering Fellowship and the Engineering Academic Scholar Certificate from the University of Michigan. He also received the Distinguished Graduate Student Masters Research Award, in 2001, from Texas A\&M University.

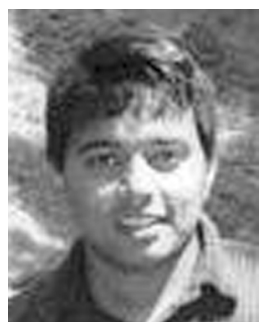

Madhusudhan Chellappa received the B.E. degree in electrical and electronics engineering from the University of Madras, Chennai, India, in 2001, and the M.S. degrees in electrical engineering and aerospace engineering from the University of Michigan, Ann Arbor, in 2003 and 2004, respectively.

Currently, he develops on-board diagnostic and feedback control algorithms for diesel engine emissions control devices for Cummins Engine Company, Columbus, IN. His areas of interest include real-time control and failure diagnostics of multivariable systems, system identification and neural networks with application to automotive and aerospace engineering industries.
Jean Luc Valk received the M.S. degree in electrical engineering from the Eindhoven University of Technology, Eindhoven, the Netherlands, in 2004.

He completed an internship at the University of Michigan, Ann Arbor, in 2003.

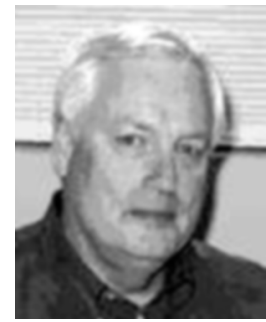

N. Harris McClamroch (M'66-F'88) received the $\mathrm{Ph} . \mathrm{D}$. degree in engineering mechanics from The University of Texas at Austin, Austin.

In 1967, he joined The University of Michigan, Ann Arbor, where he is currently a Professor in the Department of Aerospace Engineering. During the past fifteen years, his primary research interest has been in nonlinear control. He has worked on many control engineering problems arising in flexible space structures, robotics, automated manufacturing, aerospace flight systems. control technologies for buildings and bridges, and

Dr. McClamroch received the Control Systems Society Distinguished Member Award and was a recipient of the IEEE Third Millennium Medal. He has served as an Associate Editor and Editor of the IEEE TRANSACTIONS ON AUTOMATIC CONTROL, and he has held numerous positions, including President, in the IEEE Control Systems Society.

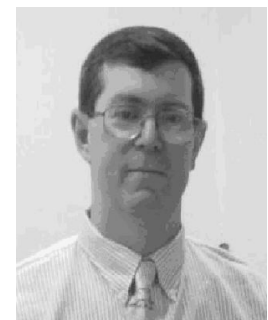

Dennis S. Bernstein (M'82-F'00) is a Professor in the Aerospace Engineering Department at the University of Michigan, Ann Arbor. His research interests are in system identification, state estimation, and adaptive control, with application to vibration and flow control and data assimilation. $\mathrm{He}$ is currently the Editor-in-Chief of the IEEE Control Systems Magazine, and he is the author of Matrix Mathematics, Theory, Facts, and Formulas with Application to Linear Systems Theory (Princeton Univ. Press, 2005). 